\title{
DETECCIÓN DEL DAÑO SÍSMICO DE UN MARCO TRIDIMENSIONAL DE CONCRETO REFORZADO MEDIANTE PRUEBAS DE VIBRACIÓN AMBIENTAL Y FORZADA
}

\author{
Jesús Valdés González ${ }^{(1)}$, Jaime De la Colina Martínez ${ }^{(1)}$ y Carlos Alberto González Pérez ${ }^{(1)}$ \\ RESUMEN
}

Se presenta el estudio experimental de un marco tridimensional de concreto reforzado de dos niveles, el cual se daño progresivamente hasta que alcanzó un nivel de daño estructural importante. El marco se sometió a diferentes etapas progresivas de carga dinámica, las cuales simulan incrementos de la intensidad del movimiento sísmico del terreno. El marco se excitó mediante un péndulo de pruebas, el cual es un dispositivo que excita la estructura fija a su plataforma mediante un movimiento armónico a lo largo de una dirección horizontal para frecuencias seleccionadas. Para cada nivel de excitación se realizaron pruebas de vibración ambiental y forzada al marco de concreto. De esta forma fue posible caracterizar su nivel de daño estructural en función de la correlación estadística entre dos funciones de transferencia $(F T)$, correspondientes a la condición inicial sin daño y a otros estados con determinado nivel de daño.

Palabras clave: daño sísmico; detección de daño; pruebas experimentales; vibración ambiental; funciones de transferencia

\section{SEISMIC DAMAGE DETECTION OF A REINFORCED CONCRETE TRIDIMENSIONAL FRAME USING AMBIENTAL AND FORCED VIBRATION TESTS}

\begin{abstract}
This paper presents an experimental study conducted on a tridimensional two-story reinforcedconcrete frame, which was progressively damaged until it reached a significant structural damage. The frame was subjected to different dynamic load stages, representatives of increasing intensities of the earthquake ground motion. The frame was excited with a testing pendulum, which is a device that excited the structure attached to the pendulum platform with a harmonic motion along one horizontal direction at selected frequencies. For each excitation level, forced and ambient vibration tests were conducted and it was possible to characterize the concrete frame damage as a function of the correlation between two transfer functions, corresponding to the initial undamaged condition of the frame and subsequent damage stages.
\end{abstract}

Keywords: seismic damage; damage detection; experimental tests; ambient vibration; transfer functions

Artículo recibido el 4 marzo de 2014 y aprobado para su publicación el 30 de julio de 2015. Se aceptarán comentarios y/o discusiones hasta cinco meses después de su publicación.

(1) Facultad de Ingeniería, Universidad Autónoma del Estado de México, Cerro de Coatepec S/N, Ciudad Universitaria, Toluca, México, C.P.50100.jvaldes@uaemex.mx jaime_delacolina@yahoo.com albertglezp@yahoo.com.mx 


\section{INTRODUCCIÓN}

Los edificios que se localizan en zonas sísmicas se ven expuestos a lo largo de su vida útil a distintos temblores que pueden afectar su integridad estructural en distintos niveles de daño. Para estos edificios es importante desarrollar técnicas y métodos que permitan obtener estimaciones confiables del posible daño causado por temblores de intensidad alta. Después de que ocurre un temblor, es importante evaluar inmediatamente el daño estructural de los edificios para tomar decisiones referentes a una posible evacuación del edificio, y/o a una posible reparación del mismo. Una primera inspección confiable es esencial en estos casos para evitar inconvenientes o incluso riesgo a los ocupantes del edificio.

Actualmente existen distintos métodos no destructivos para llevar a cabo la evaluación del daño estructural. Estos métodos se pueden clasificar en dos grupos: los convencionales y los dinámicos. Entre los métodos convencionales están: la inspección visual, la emisión acústica, el radiográfico, el de ultrasonido, entre algunos otros. Mayores referencias acerca del uso de estos métodos se pueden encontrar en (Prine, 1998; Voigt et al., 2003a y b; Wang, 2004 y Rhazi, 2006). La principal desventaja de los métodos convencionales es que su aplicación no resulta práctica, ya que se requiere conocer a priori la localización del daño y tener libre acceso a la zona de inspección, lo cual no sucede en la mayoría de los casos (Salawu y Williams, 1995). En los edificios, éste es un aspecto muy importante debido a que su estructura está generalmente cubierta por diferentes elementos no estructurales tales como plafones, techos y paredes falsas, muros divisorios, etc., lo cual dificulta en alto grado la aplicación de los métodos de inspección convencionales. Actualmente, el método de inspección más común que se utiliza en los edificios es la inspección visual. Además de los inconvenientes comentados anteriormente, el método de inspección visual requiere ser realizado por personal calificado cuyo juicio acerca del daño puede ser subjetivo y depende, en general, de la experiencia y nivel de conocimientos del inspector. La mayoría de las evaluaciones de este tipo para edificios de concreto consisten en cuantificar y medir los anchos de las grietas que se presenten en los elementos estructurales, para después relacionar esta información con un cierto nivel de daño. Con base en esta inspección visual, se toman decisiones relacionadas a la evacuación de los edificios o a la realización de estudios más detallados. El gran número de edificios que necesitan evaluarse después de un temblor intenso y el reducido número de personas calificadas para realizar este tipo de inspecciones, muestran la necesidad de cambiar la inspección convencional por métodos más confiables que puedan automatizarse.

En contraste a los métodos convencionales, los método dinámicos se basan en la medición de la vibración de la estructuras para evaluar su daño. Estos métodos se fundamentan en la hipótesis de que cualquier daño estructural representa cierta degradación de la rigidez de la estructura, lo cual modifica sus propiedades dinámicas. Bajo este enfoque, usualmente se utilizan los cambios medidos en los parámetros modales (por ejemplo: frecuencias naturales, amortiguamientos modales, formas modales, derivadas de las formas modales, etc.), para evaluar los cambios en las propiedades físicas de la estructura que pudieran indicar daño o degradación estructural. También se han estudiado los cambios de las funciones de transferencia $(F T)$ como posibles parámetros que permitan identificar el daño estructural (Roitman et al., 1992; Lew, 1995; Wang et al., 1997; James, et al., 1998; Thyagarajan et al., 1998; Sampaio et al., 1999; Lee y Shin, 2002).

En la bibliografía citada, se les llama funciones de respuesta en frecuencia $(F R F s)$ a las funciones que en este trabajo se les denomina funciones de transferencia $(F T)$. Las $F T$ parecen ser un estimador más confiable para detectar el daño estructural, en comparación a los parámetros modales. Esto se debe a que las FT se obtienen directamente a partir de los datos registrados (mediciones de vibración), por lo que su margen de error sólo se limita a un posible error en las mediciones. Por su parte, los parámetros modales se extraen indirectamente de los datos registrados, a partir de procedimientos cuya aplicación puede generar cierto error adicional al de las mediciones. En este sentido, es más razonable utilizar las FT para 
identificar el daño estructural, particularmente en aquellas estructuras cuyos modos se encuentran muy próximos entre sí (Ni et al., 2006). La precisión de los métodos de identificación de daño que se basan en las $F T$ depende en gran medida del intervalo de frecuencias que se seleccione.

Los métodos dinámicos parecen ser uno de los procedimientos más prácticos para la detección de daño, ya que tienen la ventaja de no requerir información a priori acerca de la localización del daño, y tampoco requieren libre acceso a la zona de inspección. Se han llevado a cabo distintos estudios relacionados con métodos dinámicos de detección de daño estructural, cuyos resultados muestran las ventajas que tiene su uso. Doebling et al. (1996) presentan una extensa descripción de varios métodos dinámicos y una revisión bibliográfica referente a ellos. El futuro de los métodos dinámicos es promisorio, pero aún quedan muchos aspectos por resolver antes de que puedan utilizarse de manera generalizada y confiable.

Este artículo presenta el estudio de laboratorio de un marco tridimensional de concreto reforzado de dos niveles, el cual se dañó de manera progresiva hasta que alcanzó un daño estructural severo. A diferencia de otros estudios similares en los cuales el daño estructural se ha provocado de manera artificial, en este caso el marco se sometió a distintas etapas progresivas de carga dinámica representativas de incrementos de la intensidad sísmica del movimiento del suelo. Esto se llevó a cabo mediante el uso de un péndulo de prueba, el cual es un dispositivo que permitió excitar el modelo colocado en su plataforma mediante un movimiento armónico a lo largo de una dirección horizontal para ciertas frecuencias seleccionadas. Con este dispositivo fue posible inducir distintos niveles de daño estructural al marco mediante excitaciones en la base. Después de cada nivel de daño alcanzado se realizaron mediciones de vibración ambiental y pruebas de vibración forzada con base en las cuales se identificaron las propiedades dinámicas del modelo y se calcularon sus $F T$. El objetivo del estudio fue caracterizar el nivel de daño del modelo en función de la variación de algunas características relacionadas a su respuesta dinámica. Para ello se analizaron registros de su respuesta obtenidos, tanto en pruebas de vibración ambiental, como forzada. Con base en los resultados fue posible caracterizar el nivel de daño estructural del modelo en términos de la correlación entre las $F T$ correspondientes a un estado inicial sin daño y a otro estado con determinado nivel de daño. De igual forma se observaron cambios importantes en la primera frecuencia modal del marco, conforme aumentó su nivel de daño.

\section{PÉNDULO DE PRUEBA}

El péndulo de prueba que se utilizó en los experimentos está formado por una plataforma de acero que cuelga del techo del laboratorio mediante cuatro tirantes de acero. Sobre la plataforma se tiene sujeto un excitador de masas excéntricas con eje vertical de rotación (figura 1), el cual le transmite a la plataforma del péndulo una fuerza pseudo - armónica en la dirección longitudinal. Esta fuerza se puede aplicar con diferentes frecuencias y amplitudes, aunque para cada prueba, tanto la frecuencia como la amplitud permanecen constantes. El mecanismo hace que durante una prueba la plataforma del péndulo presente un movimiento longitudinal armónico, el cual se transmite al modelo de concreto que se encuentra sobre la plataforma, tal como si fuese el movimiento sísmico del suelo que se presenta durante un temblor. En la figura 2 se presentan en forma esquemática la geometría del péndulo y del marco de concreto ensayado, en los cuales se identifican sus principales componentes. En la figura 3 se muestra una imagen del péndulo y el marco. 


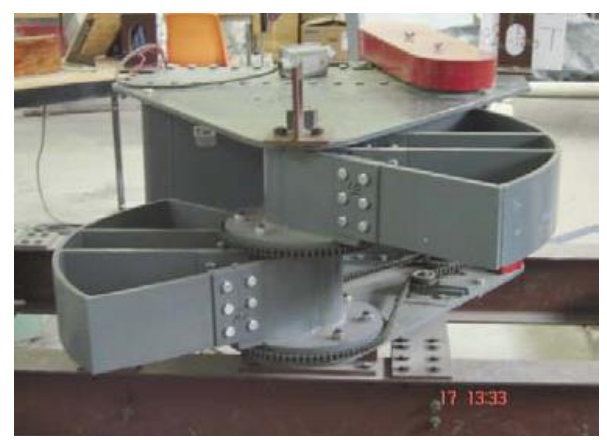

Figura 1. Excitador de masas excéntricas sujeto a la plataforma del péndulo de prueba

El periodo natural del sistema completo (péndulo y marco de concreto) fue cercano a los $5 \mathrm{~s}$. Este periodo se considera lo suficientemente largo, comparado con los periodos modales del marco solo, de tal forma que fue posible considerar que ambos sistemas estaban virtualmente desacoplados. El periodo del marco solo correspondiente al primer modo para el estado sin daño de la estructura fue de $T_{1}=0.312 \mathrm{~s}\left(f_{1}\right.$ $=3.2 \mathrm{~Hz})$ y varió hasta $T_{l}=0.438 \mathrm{~s}\left(f_{l}=2.28 \mathrm{~Hz}\right)$ para la última etapa de daño que alcanzó el modelo. En párrafos posteriores se describe con detalle la variación de dicho periodo (frecuencia) durante los distintos estados de daño del modelo. Durante las pruebas, sólo se permitió el movimiento longitudinal de la plataforma del péndulo. En el trabajo de De la Colina y Valdés (2010) se puede consultar mayor información referente al péndulo de prueba.

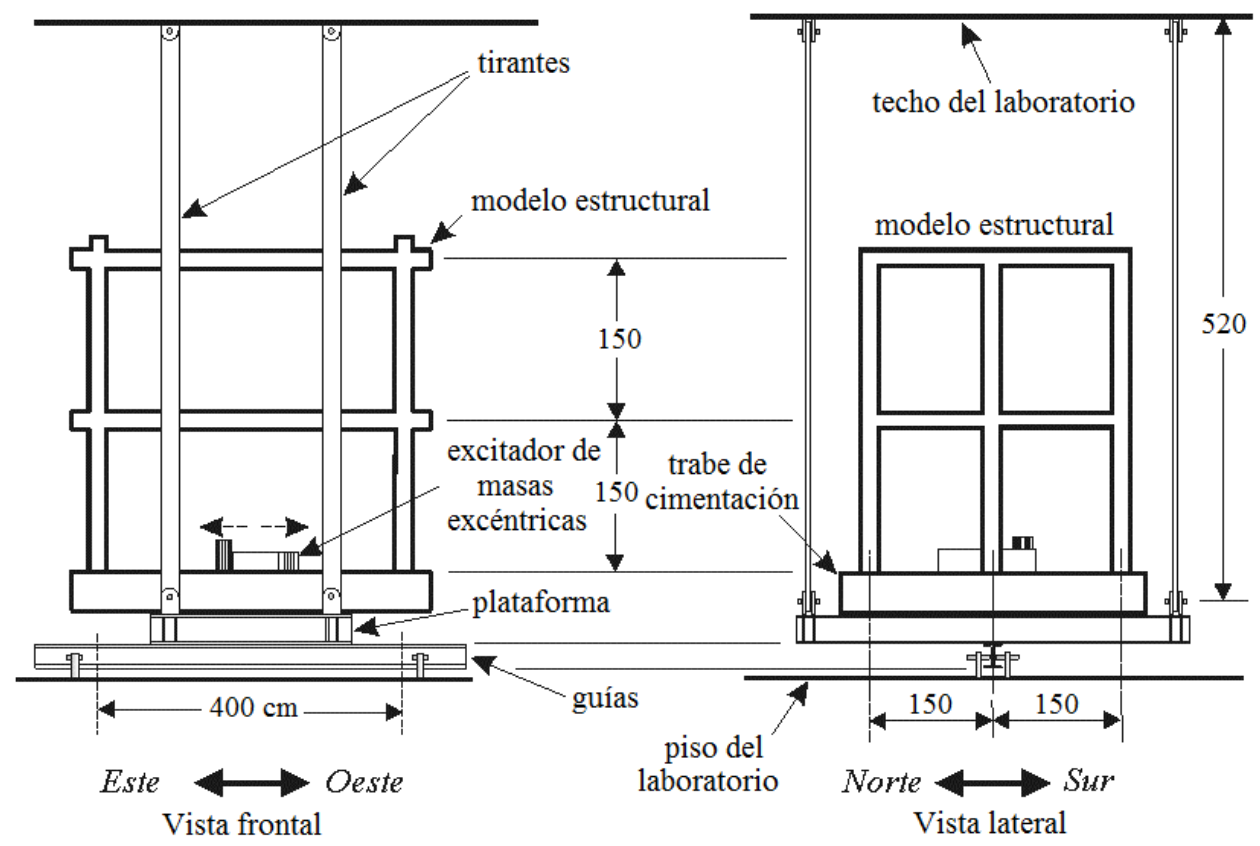

Figura 2. Vistas frontal y lateral del péndulo de prueba junto con el modelo de concreto

\section{MODELO EXPERIMENTAL}

El modelo de concreto reforzado que se utilizó en este estudio correspondió a un marco tridimensional de dos niveles, formado por trabes y columnas. Las dimensiones en planta del modelo 
fueron de $400 \mathrm{~cm}$ en la dirección $X$ y $300 \mathrm{~cm}$ en la dirección $Y$. En la dirección $X$ el modelo tuvo una crujía y en la dirección $Y$ dos crujías. La altura de cada uno de los dos niveles fue de $150 \mathrm{~cm}$, por lo que su altura total fue de $300 \mathrm{~cm}$. La dirección de la aplicación de la fuerza del excitador y del movimiento de la plataforma del péndulo correspondió a la dirección $X$. El modelo no se construyó con losas, pero se colocaron tensores de acero para tratar de simular un comportamiento de diafragma rígido. La cimentación del marco estuvo formada por trabes de concreto reforzado, las cuales se apoyaron y sujetaron a la plataforma del péndulo mediante anclas de acero. Las columnas del modelo se anclaron directamente a las trabes de la cimentación. La figura 4 muestra las dimensiones en planta del modelo (cimentación, primer y segundo nivel). En la figura 5 se muestran las dimensiones, los detalles del refuerzo y las propiedades de los materiales de los elementos estructurales del marco.

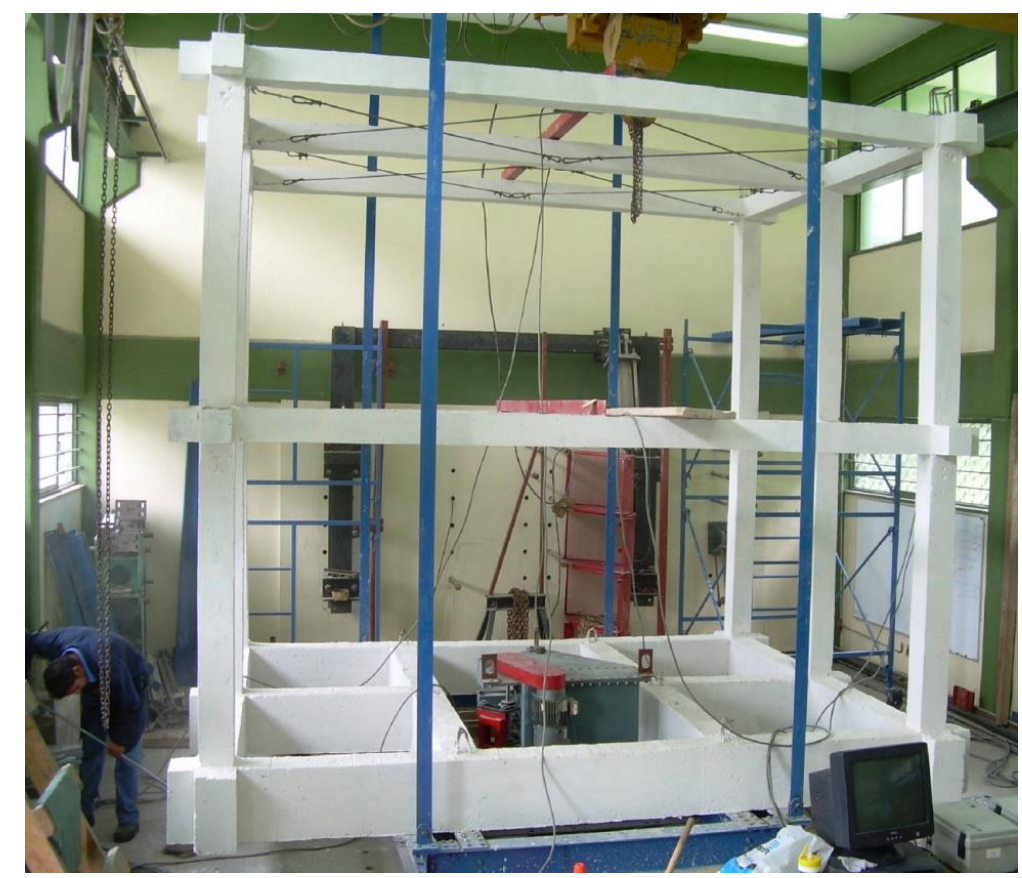

Figura 3. Péndulo de prueba junto con el modelo de concreto reforzado que se estudia

Los armados de las trabes y columnas se obtuvieron suministrando la cantidad de acero necesaria para resistir las demandas estimadas mediante el análisis convencional de un modelo elástico del marco de prueba. Para ello se supuso la acción de cargas laterales equivalentes a una fuerza cortante en la base del modelo igual a una tercera parte, aproximadamente, de la máxima fuerza cortante que se podría generar con el péndulo de pruebas. De esta forma, se cubrió la posibilidad de que el modelo presentara una resistencia mayor a la estimada bajo ese nivel de fuerza cortante, y se tuviese un margen que permitiera aplicarle una mayor fuerza hasta alcanzar su resistencia durante las pruebas realizadas. Las resistencias se calcularon a partir de los criterios establecidos en el Reglamento de Construcciones del DF (NTCC-2004). En la Tabla 1 se presentan las resistencias nominales a flexión y cortante de las trabes y columnas del marco de prueba, así como las máximas demandas que se alcanzaron durante las pruebas realizadas $(N=1$ a 15). Se observa que la máxima demanda a flexión de las trabes se alcanzó durante la prueba 6 , la cual fue superior a la capacidad nominal resistente de la sección. Por lo que se refiere a las columnas, la máxima demanda a flexión se presentó en la última prueba $(N=15)$, la cual fue inferior a la capacidad nominal resistente de la sección. Esto demuestra que la falla del modelo se alcanzó debido a la falla a flexión de las trabes, mientras que las columnas permanecieron sin daño (columna fuerte - trabe débil). También se observa que la resistencia nominal a cortante del concreto $\left(V_{C R}\right)$, tanto para trabes como para 
columnas, fue superior a las máximas demandas que se alcanzaron; lo cual demuestra que no se presentó ninguna falla por cortante. Los efectos de la carga axial en las columnas fueron tan bajos, que no se requirió detallar el refuerzo transversal de las columnas por confinamiento.

Tabla 1. Demandas y capacidades de trabes y columnas

\begin{tabular}{ccccc}
\hline \multirow{2}{*}{ Elemento } & \multicolumn{2}{c}{ Flexión } & \multicolumn{2}{c}{ Cortante } \\
\cline { 2 - 5 } & $\begin{array}{c}\text { Capacidad } \\
M_{n}(\mathrm{~kg}-\mathrm{cm})\end{array}$ & $\begin{array}{c}\text { Máxima Demanda } \\
M(\mathrm{~kg} / \mathrm{cm})\end{array}$ & $\begin{array}{c}\text { Capacidad } \\
V_{C R}(\mathrm{~kg})\end{array}$ & $\begin{array}{c}\text { Máxima Demanda } \\
V(\mathrm{~kg})\end{array}$ \\
\hline Trabe & 12466 & $12540(N=6)$ & 464.46 & $60(N=6)$ \\
Columna & 84275 & $71500(N=15)$ & 913 & $290.78(N=15)$ \\
\hline
\end{tabular}

En una estructura con diafragma rígido las fuerzas cortantes que toman los marcos estructurales son proporcionales a sus rigideces, mientras que en una estructura con diafragma flexible las fuerzas cortantes que toman los marcos son proporcionales a su masa inercial tributaria. El modelo ensayado presenta un comportamiento intermedio entre ambos casos, ya que si bien, los tensores que se utilizaron para simular el diafragma no tienen una rigidez infinita que evite la completa distorsión en el plano horizontal de los tableros de cada nivel, sí tienen cierta rigidez que minimiza esta distorsión. En general, durante las pruebas se pudo observar que los marcos se dañaron de manera uniforme entre sí, lo cual se puede atribuir a que las masas inerciales fueron muy similares entre los tres marcos, además de que el sistema de tensores proporcionó cierta rigidez a los tableros de cada nivel logrando simular en cierta medida un diafragma semirrígido. Para interpretar los resultados de las pruebas que se realizaron al modelo, no fue necesario suponer un comportamiento dado del diafragma para interpretar los resultados, ya que estos son inherentes al modelo ensayado dadas sus características propias.

El efecto que tuvo el que los marcos extremos alineados en la dirección $Y$ no se encontraran apoyados directamente sobre la plataforma del péndulo, sino sobre las trabes de cimentación, fue mínimo. Esto se debió a que las trabes de cimentación fueron lo suficientemente rígidas a flexión y cortante para evitar que en el volado tan corto que tenían pudiesen presentarse deflexiones importantes que favorecieran algún tipo de cabeceo del modelo.

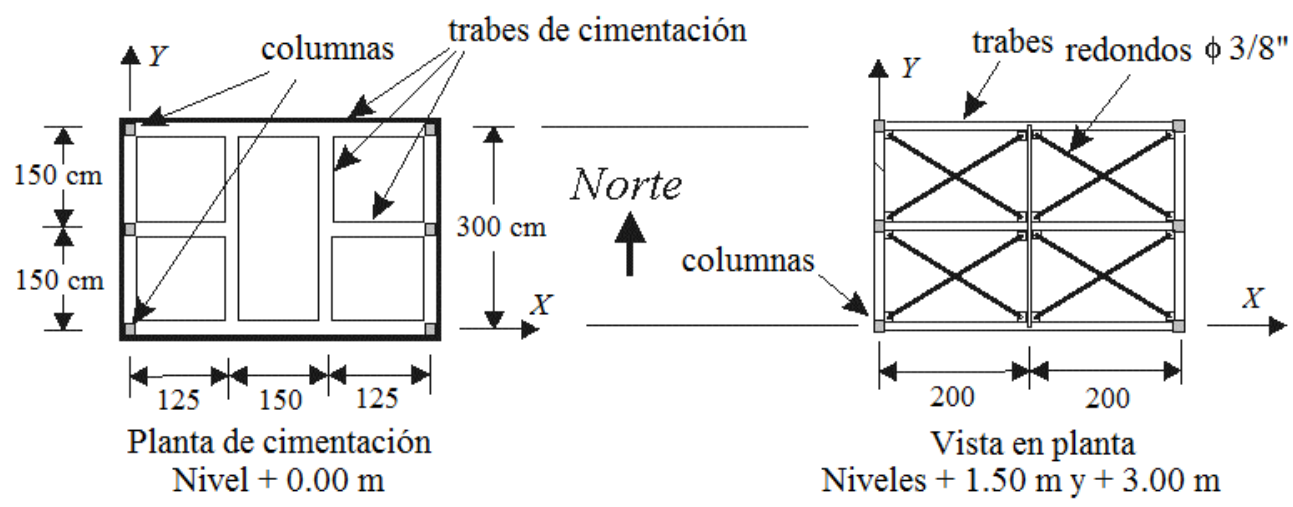

Figura 4. Dimensiones en planta del modelo (cimentación y vigas de niveles) 


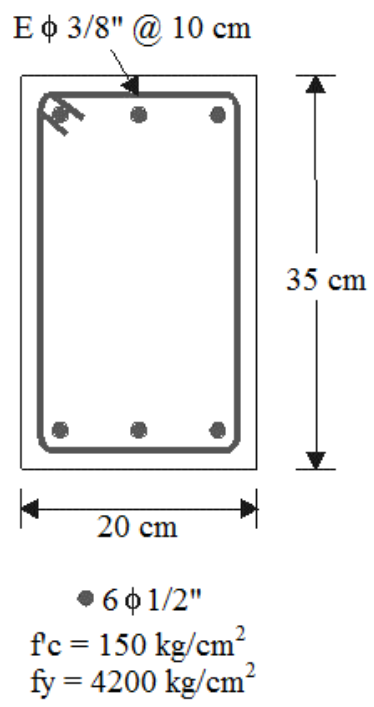

Trabes de cimentación

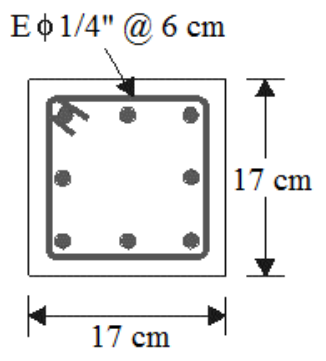

- $8 \phi 3 / 8^{\prime \prime}$

$\mathrm{f}^{\prime} \mathrm{c}=150 \mathrm{~kg} / \mathrm{cm}^{2}$ fy $=4200 \mathrm{~kg} / \mathrm{cm}^{2}$

Columnas

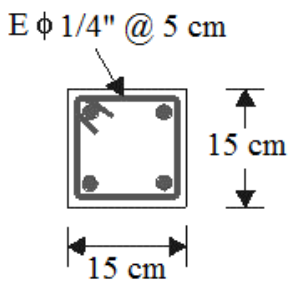

- $4 \phi 1 / 4 "$

$\mathrm{f}^{\prime} \mathrm{c}=150 \mathrm{~kg} / \mathrm{cm}^{2}$

$\mathrm{fy}=2100 \mathrm{~kg} / \mathrm{cm}^{2}$

Trabes

Figura 5. Detalles del refuerzo de las trabes, columnas y trabes de cimentación del modelo, con sus correspondientes propiedades de materiales

La respuesta dinámica del modelo se registró mediante cinco acelerómetros colocados de la siguiente forma. Uno en el centro de la plataforma del péndulo (Nivel $+0.00 \mathrm{~m}$ ), dos en el primer nivel del modelo (Nivel $+1.50 \mathrm{~m}$ ) y dos en el segundo nivel (Nivel $+3.00 \mathrm{~m}$ ). La figura 6 muestra la localización de los acelerómetros, la dirección en la cual se registraron las señales y el número asociado a cada canal de registro.

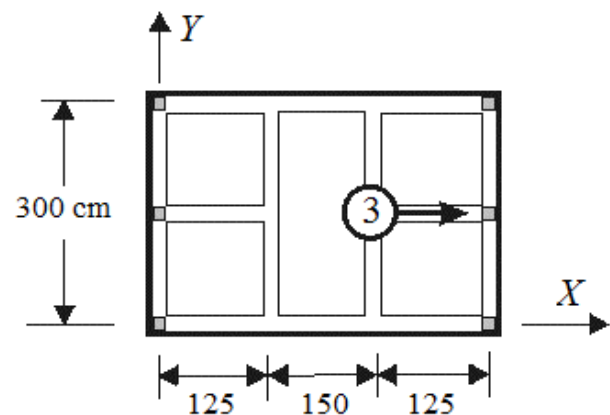

Nivel $+0.00 \mathrm{~m}$

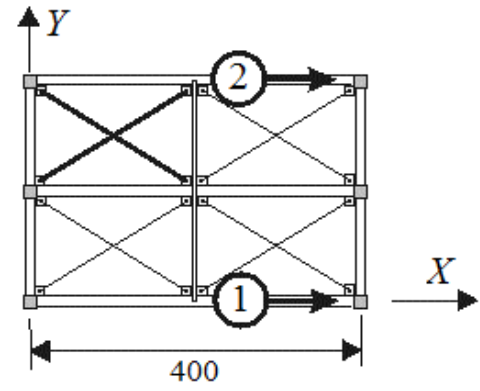

Nivel $+1.50 \mathrm{~m}$

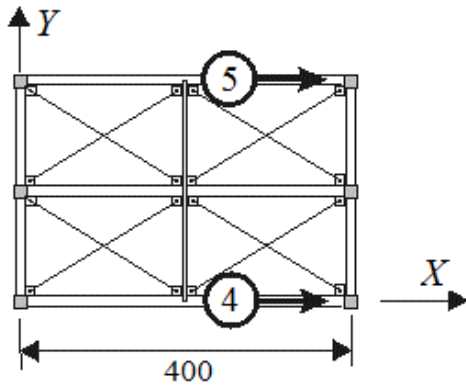

Nivel $+3.00 \mathrm{~m}$

Figura 6. Localización de los acelerómetros que registraron la vibración del modelo (canales de registro)

El marco de concreto reforzado que se utilizó en las pruebas, se diseño de la misma forma como se diseña la estructura de cualquier edificio real, y su respuesta se analizó en términos de su propio comportamiento. El marco se concibió como una estructura real, y las pruebas se llevaron a cabo para obtener un entendimiento cualitativo de la detección del daño en estructuras de edificios similares a ésta y bajo el mismo tipo de condiciones. No se trata de extrapolar resultados cuantitativos a alguna otra estructura en particular. Debido a que el marco que se probó no representa algún prototipo en particular, no fue necesario establecer requerimientos de escalamiento y similitud en su diseño. 


\section{ETAPAS DE DAÑO}

Mediante pruebas de vibración forzada se indujeron quince etapas de daño al modelo $(N=1$ a 15$)$. Cada una correspondió a una prueba en específico para una frecuencia de operación y cierta masa rotacional del excitador. La masa rotacional se incrementó progresivamente de una prueba a otra de manera constante. Debido a que la amplitud de la fuerza inducida por el excitador a la plataforma del péndulo depende tanto de la masa rotacional como de la frecuencia de operación, la amplitud de la fuerza que induce el excitador a la plataforma del péndulo varió de una prueba a otra de manera creciente y progresiva. Las frecuencias de operación del excitador fueron $f=3.0 \mathrm{~Hz}$ para las pruebas 1 a $6, f=2.3 \mathrm{~Hz}$ para las pruebas 7 y $8, f=2.1 \mathrm{~Hz}$ para las pruebas 9 a 13 y $f=2.0 \mathrm{~Hz}$ para las pruebas 14 y 15 . Esta variación de frecuencias se seleccionó para generar incrementos de la fuerza inducida por el excitador, aproximadamente constantes.

Después de cada prueba de vibración forzada que generó un determinado nivel de daño estructural en el modelo, se llevó a cabo una prueba de vibración ambiental. Las pruebas de vibración ambiental consistieron en registrar durante dos minutos la vibración del sistema (péndulo + modelo) ocasionado solamente por el tráfico y las actividades humanas de los alrededores del laboratorio. Debido a que el modelo estaba dentro del laboratorio, el viento no lo excitó durante estas pruebas.

En realidad ambos tipos de prueba, vibración forzada y vibración ambiental, son pruebas de vibración forzada. Los dos tipos de pruebas son iguales en el sentido de que en ambos casos la excitación al marco de concreto fue causada por la vibración de la plataforma del péndulo. Las diferencias entre ellas son la amplitud y el contenido de frecuencias de la vibración de la plataforma del péndulo. Para distinguir entre la vibración causada por el excitador de masas excéntricas, de la vibración causada por el tráfico y las actividades humanas en los alrededores del laboratorio, al segundo tipo se les denomina pruebas de vibración ambiental.

Todos los registros de las aceleraciones obtenidos durante las pruebas de vibración ambiental y forzada, fueron corregidos por línea base y filtrados de acuerdo a las recomendaciones de Chiu (1997). A partir de los registros procesados se calcularon velocidades y desplazamientos. La figura 7 muestra algunos registros típicos de aceleraciones y desplazamientos que se obtuvieron de las pruebas de vibración forzada llevadas a cabo $(N=8)$.
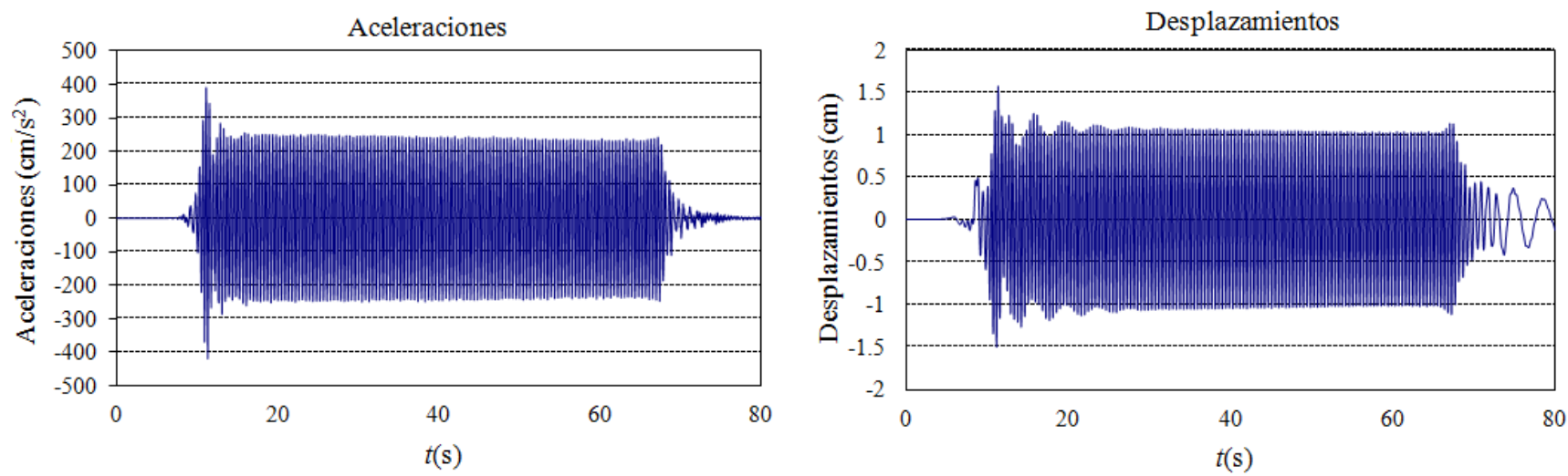

Figura 7. Registros de aceleraciones y desplazamientos correspondientes al canal 5 para la prueba $N=8$ ( $t$ denota tiempo) 
Para caracterizar el nivel de daño correspondiente a cada prueba de vibración forzada, se obtuvo la máxima distorsión global del modelo $\left(\gamma_{\max }\right)$ que se presentó a lo largo de toda la duración de los registros. La cual se calculó de acuerdo a la siguiente ecuación

$\gamma_{\max }=\max \left[\frac{x_{2}(t)-x_{B}(t)}{H}\right]$

donde $x_{2}$ es el desplazamiento total del segundo nivel del modelo en el instante $t, x_{B}$ el desplazamiento de su base (plataforma del péndulo, canal 3) en el mismo instante $t$ y $H$ la altura total del modelo. $x_{2}$ se calculó como el promedio de los desplazamientos correspondientes a los canales 4 y 5 . Todos estos cálculos se hicieron para el estado estable de la respuesta.

La figura 8 muestra los valores de la máxima fuerza cortante basal ( $V_{\max }$ ) que actuó en el marco durante el estado estable de cada prueba de vibración forzada, en función de la máxima distorsión global del modelo $\left(\gamma_{\max }\right)$ que se alcanzó en la prueba correspondiente. La fuerza cortante basal se calculó de acuerdo a la ec. 2. Esta ecuación satisface el equilibrio dinámico del modelo despreciando el amortiguamiento.

$V=\ddot{x}_{2} m_{2}+\ddot{x}_{1} m_{1}$

donde $\ddot{x}_{2}$ es la aceleración total del segundo nivel del modelo, $\ddot{x}_{1}$ la aceleración total del primer nivel y $m_{2}$ y $m_{l}$ las masas traslacionales de inercia del segundo y primer nivel respectivamente.

Las aceleraciones $\ddot{x}_{2}$ y $\ddot{x}_{1}$ se calcularon como el promedio de las aceleraciones correspondientes a los canales 4 y 5 , y 1 y 2 , respectivamente. Las masas $m_{1}=1388 \mathrm{~kg}$ y $m_{2}=1180 \mathrm{~kg}$ se calcularon tomando en cuenta la masa de las trabes y columnas correspondientes a cada nivel.

Con base en el método del decremento logarítmico propuesto en la literatura (Clough y Penzien, 1993) se hizo una estimación de la fracción de amortiguamiento crítico del modelo sin daño para una prueba de vibración libre, la cual fue cercana a $2 \%$. Dado este nivel de amortiguamiento no se esperan errores importantes en la estimación de $V$ al despreciar las fuerzas de amortiguamiento en la ec. 2.

La segunda escala vertical que se muestra en el lado derecho de la gráfica de la figura 8 corresponde al valor de $c=V_{\max } / W_{t}$, donde $W_{t}=2568 \mathrm{~kg}$ es el peso total de la estructura asociado a los grados de libertad del desplazamiento horizontal del primer y segundo nivel del modelo en la dirección $X, x_{1}$ y $x_{2}$, respectivamente. $c$ es análogo al denominado "coeficiente sísmico".

En la figura 8 se observa que el modelo permaneció bajo comportamiento elástico sin daño mayor durante las primeras seis pruebas $(N=1$ a 6$)$, las cuales correspondieron a valores de $\gamma_{\max }<0.0025$ aproximadamente. Las primeras grietas se detectaron visualmente después de la prueba $N=4\left(\gamma_{\max }=\right.$ 0.0018). De acuerdo al comportamiento observado, el punto de fluencia del modelo se ubicó en $\gamma_{\max }=$ 0.0025. Mediante el uso de un modelo analítico, se corroboró que el punto de fluencia del modelo se encontraba cercano a este valor. Por lo tanto, se puede establecer que la etapa de fluencia del modelo comenzó a partir de la prueba $N=7$. Tomando en cuenta que la máxima distorsión global del modelo estuvo cercana a $\gamma_{\max }=0.015$, entonces la demanda de ductilidad del modelo se puede considerar como $\mu$ = 6.0, aproximadamente. Debido a que el modelo se diseñó bajo el criterio de trabe débil y columna fuerte, la etapa de fluencia correspondió al caso en el cual las trabes del modelo iniciaron su fluencia por flexión. Las columnas permanecieron sin daño aparente durante todas las pruebas. 


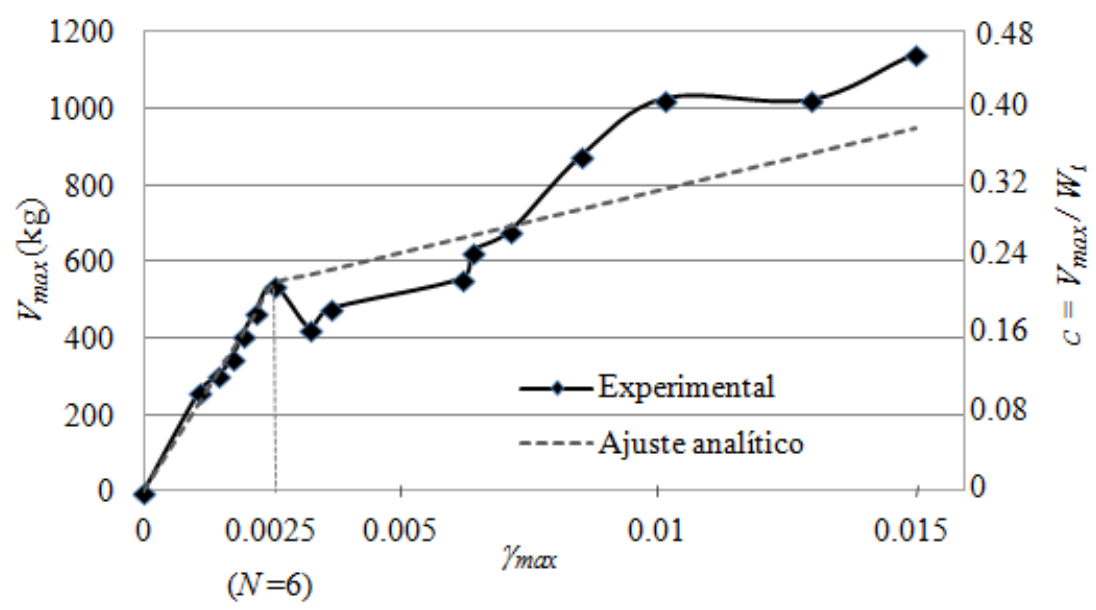

Figura 8. Relación entre la máxima fuerza cortante basal $\left(V_{\max }\right)$ y la máxima distorsión global del modelo $\left(\gamma_{\max }\right)$ para el estado estable de la vibración durante las pruebas de vibración forzada

El comportamiento de las columnas del marco durante las pruebas realizadas, las cuales permanecieron sin mayor daño aparente, se explica en la medida que la carga axial que soportaron fue muy baja, de hecho sólo soportaron el peso propio del modelo. Este comportamiento fue el mismo para todas las columnas, inclusive para las bases de las columnas del primer nivel. Los reglamentos de diseño tratan de garantizar un adecuado comportamiento sísmico de las estructuras, fomentando el diseño de estructuras que tengan trabes "débiles" y columnas "fuertes". Esto lo hacen estableciendo límites, tanto para las cargas axiales que pueden tomar las columnas, como para los momentos resistentes de las vigas y columnas que convergen a un mismo nodo. El marco de concreto se diseño cumpliendo con estos estándares, lo que provocó que el daño se concentrara en sus trabes y no en sus columnas. En general, las columnas de un marco deberían articularse cuando se forma un mecanismo de colapso. Sin embargo, en este caso no se llegó a formar un mecanismo plástico de colapso, ya que la carga lateral estuvo limitada a la capacidad del péndulo de pruebas; de ahí que sólo se hayan articulado las trabes del marco.

La gráfica de la figura 8 muestra un comportamiento consistente con las curvas de los lazos de histéresis observadas en pruebas experimentales típicas (Martínez y Wakabayashi, 1990). Este comportamiento se caracteriza por una caída súbita de la carga aplicada justo al inicio de la etapa inelástica, seguida de una recuperación posterior, tanto de rigidez como de resistencia.

La figura 9 muestra las gráficas de los lazos de histéresis para algunas de las pruebas de vibración forzada llevadas a cabo en el marco de concreto. En cada prueba se identificó la rigidez inicial del marco como porcentaje de la rigidez del marco para el caso sin daño $(N=1)$. Estos valores se muestran en cada una de las gráficas de la figura 9. Las curvas de histéresis muestran la relación entre el cortante basal que actúa en el marco de concreto $(V)$ y el desplazamiento del nivel $2\left(\Delta_{2}\right)$ dividido entre su altura $(H)$. Éste es un índice global que permite tener una buena idea del comportamiento histerético del marco durante las etapas progresivas de daño.

Se observa una reducción de la rigidez del marco durante la etapa elástica de su comportamiento ( $N$ $=6)$ cercana a $9 \%$. Una vez que el modelo ingresa a la etapa inelástica $(N=7)$ se observa una caída súbita de la rigidez cercana a $40 \%$. Para la última prueba $(N=15)$, el marco acumula una caída de rigidez de $60 \%$ aproximadamente. Durante la etapa inelástica se observó una importante variación en el decremento 
de la rigidez del modelo. La alta reducción de la rigidez del marco durante las pruebas se debe en gran medida, a su bajo nivel de redundancia.
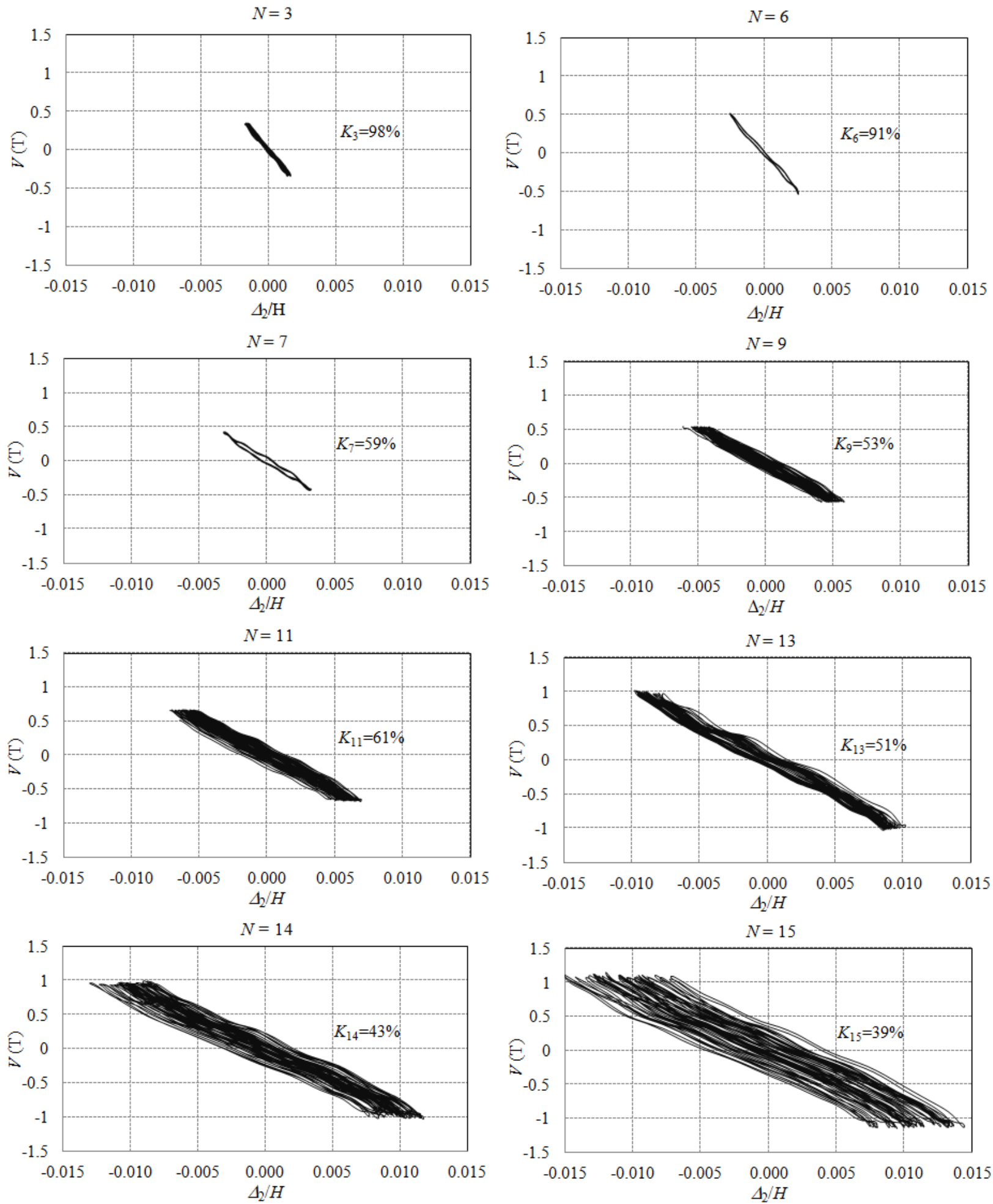

Figura 9. Curvas de histéresis para algunas de las pruebas de vibración forzada 
En general, el área que se observa dentro de los lazos de histéresis de las pruebas es pequeña, lo cual representa un bajo nivel de energía disipada en la mayoría de las pruebas. Esto se explica debido a que el experimento se planeó para dañar en forma progresiva el marco de concreto. Se intento dañar al marco muy poco en cada una de las pruebas, para que al final de las 15 pruebas realizadas, el modelo acumulara un daño estructural severo. También se aprecia que el daño que se indujo al modelo no fue el mismo durante todas las pruebas. El mayor daño que se causó al marco ocurrió durante la última prueba $(N=15)$, en cuyo caso la demanda de ductilidad del modelo fue de 6 aproximadamente. La figura 8 trata de representar la curva envolvente de los lazos de histéresis que el modelo habría tenido en caso de que el daño hubiese sido continuo y no progresivo, como en realidad fue.

La figura 10 muestra algunas de las grietas que se presentaron en las trabes del modelo durante las distintas etapas de daño.
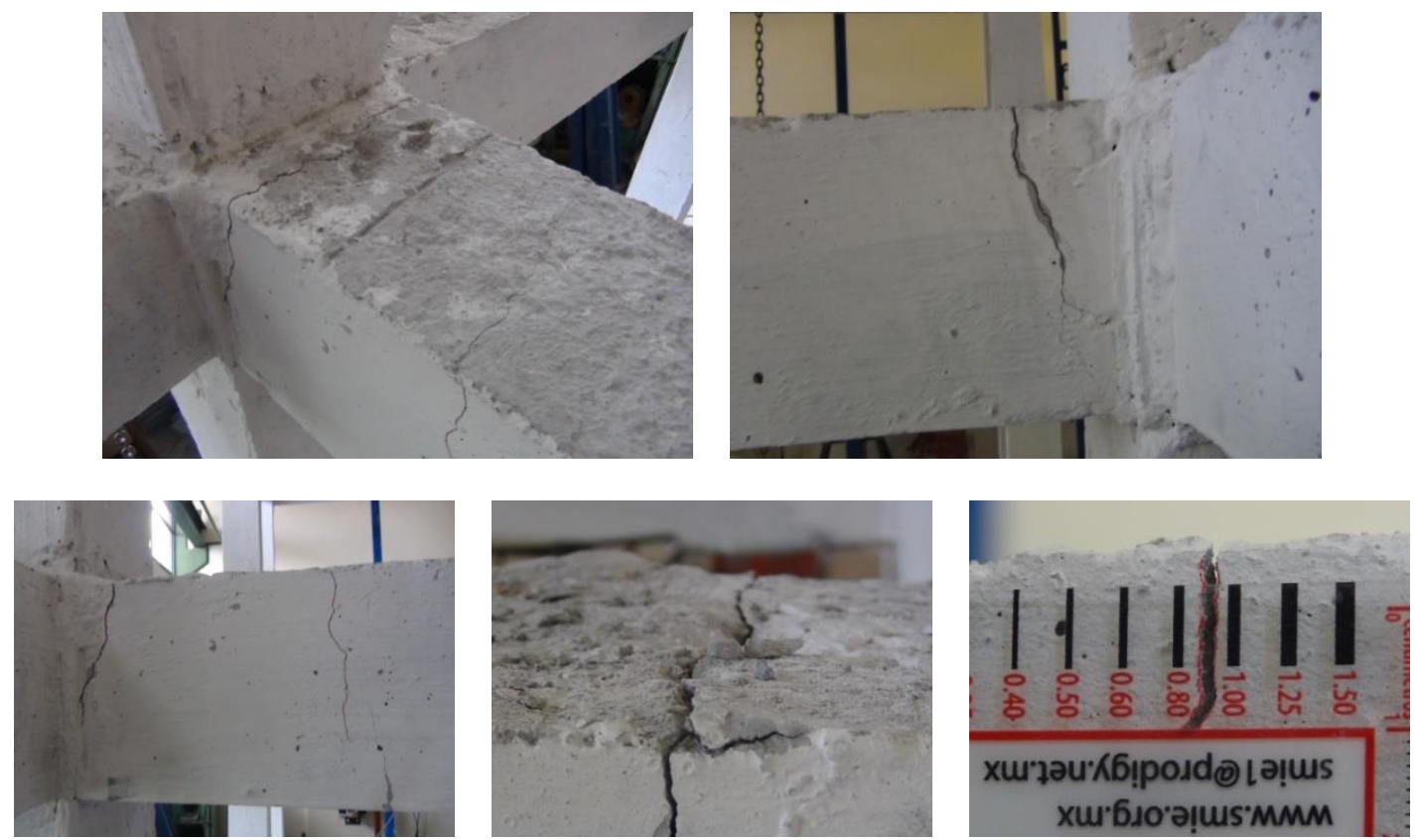

a) Prueba $N=6, \gamma_{\max }=0.0025$ (inicio de fluencia)
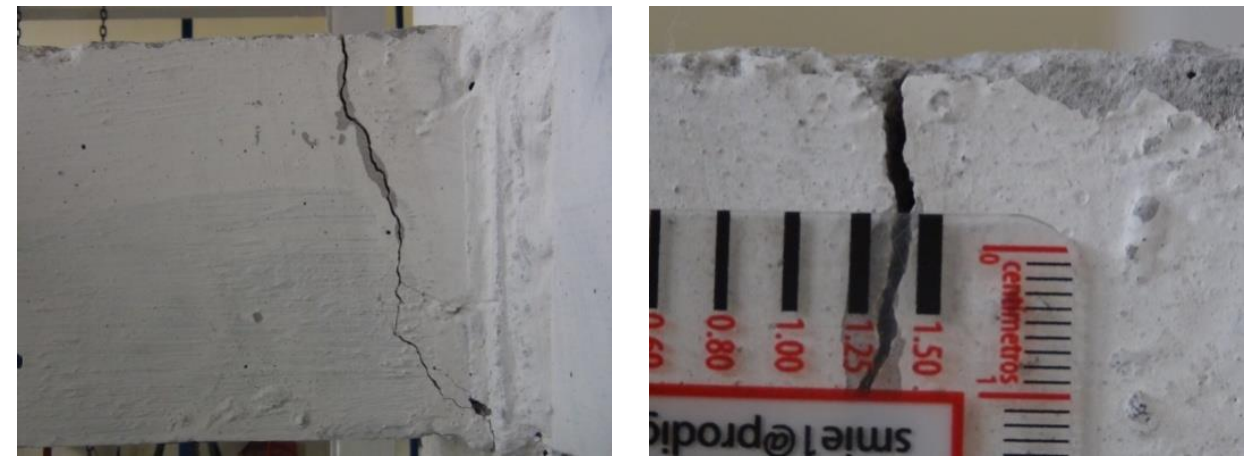

b) Prueba $N=13, \gamma_{\max }=0.01$

Figura 10. Grietas en las trabes del modelo para distintas etapas de daño 


\section{IDENTIFICACIÓN DE PROPIEDADES DINÁMICAS}

Con base en las aceleraciones registradas durante las pruebas de vibración ambiental y forzada, se identificaron para cada etapa de daño las propiedades dinámicas del modelo. Se analizaron en particular, la primera frecuencia de traslación a lo largo de la dirección $\mathrm{X}$, denotada como $f_{1}$ y su correspondiente forma modal. La identificación se realizó por medio de las funciones de transferencia $(F T)$, las cuales se calcularon como el cociente de los espectros de amplitudes de Fourier correspondientes a los canales de entrada y salida. Se seleccionó como canal de salida, el canal 5 localizado en el segundo nivel del modelo, y como canal de entrada, el canal 3 localizado en la base del modelo (plataforma del péndulo). Bajo esta definición, las $F T$ que se calcularon representan la amplificación de la vibración del marco en relación a la vibración de su base (plataforma del péndulo).

Las $F T$ tiene la ventaja de eliminar de la respuesta de la estructura la contaminación atribuida a la excitación. Por ello, las $F T$ son más confiables para identificar las propiedades dinámicas de la estructura en comparación a los espectros de amplitudes de Fourier obtenidos directamente de las aceleraciones registradas en distintos puntos de la estructura. Esto es especialmente importante en pruebas de vibración ambiental, ya que en este tipo de pruebas se tienen vibraciones de muy baja amplitud, por lo que es muy probable que la contaminación de la excitación afecte de manera importante a la vibración que se registre como respuesta de la estructura. En forma ilustrativa, se muestran en las figuras 11 y 12 los espectros de amplitudes de Fourier correspondientes a los canales 5 y 3 con sus correspondientes $F T$ para una prueba en particular $(N=3)$. La figura 11 muestra los resultados para la prueba de vibración ambiental y la figura 12 para la prueba de vibración forzada.
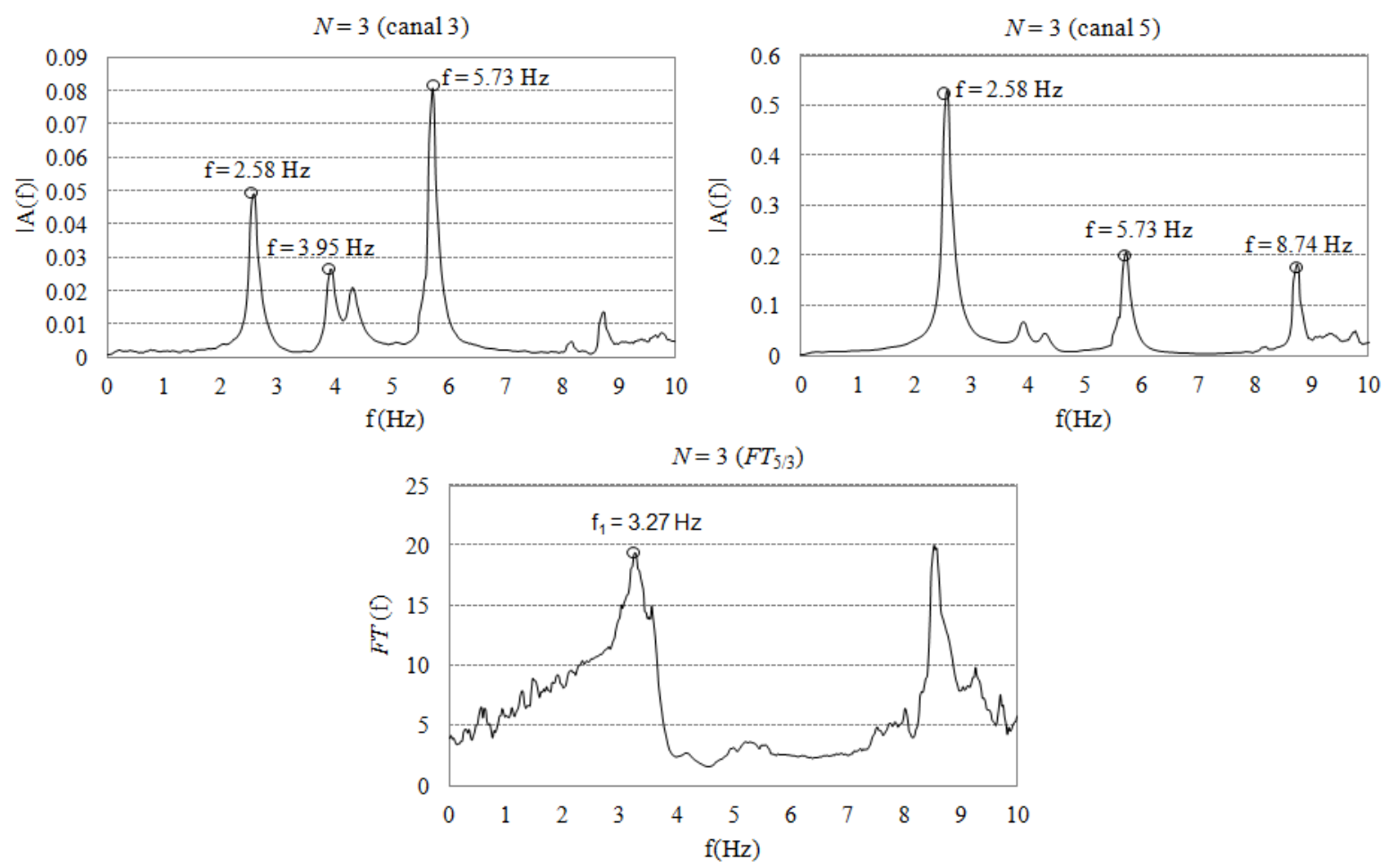

Figura 11. Espectros de amplitudes de Fourier de los canales 3 (entrada) y 5 (salida) con su correspondiente $F T$ obtenidos de una prueba de vibración ambiental $(N=3)$ 

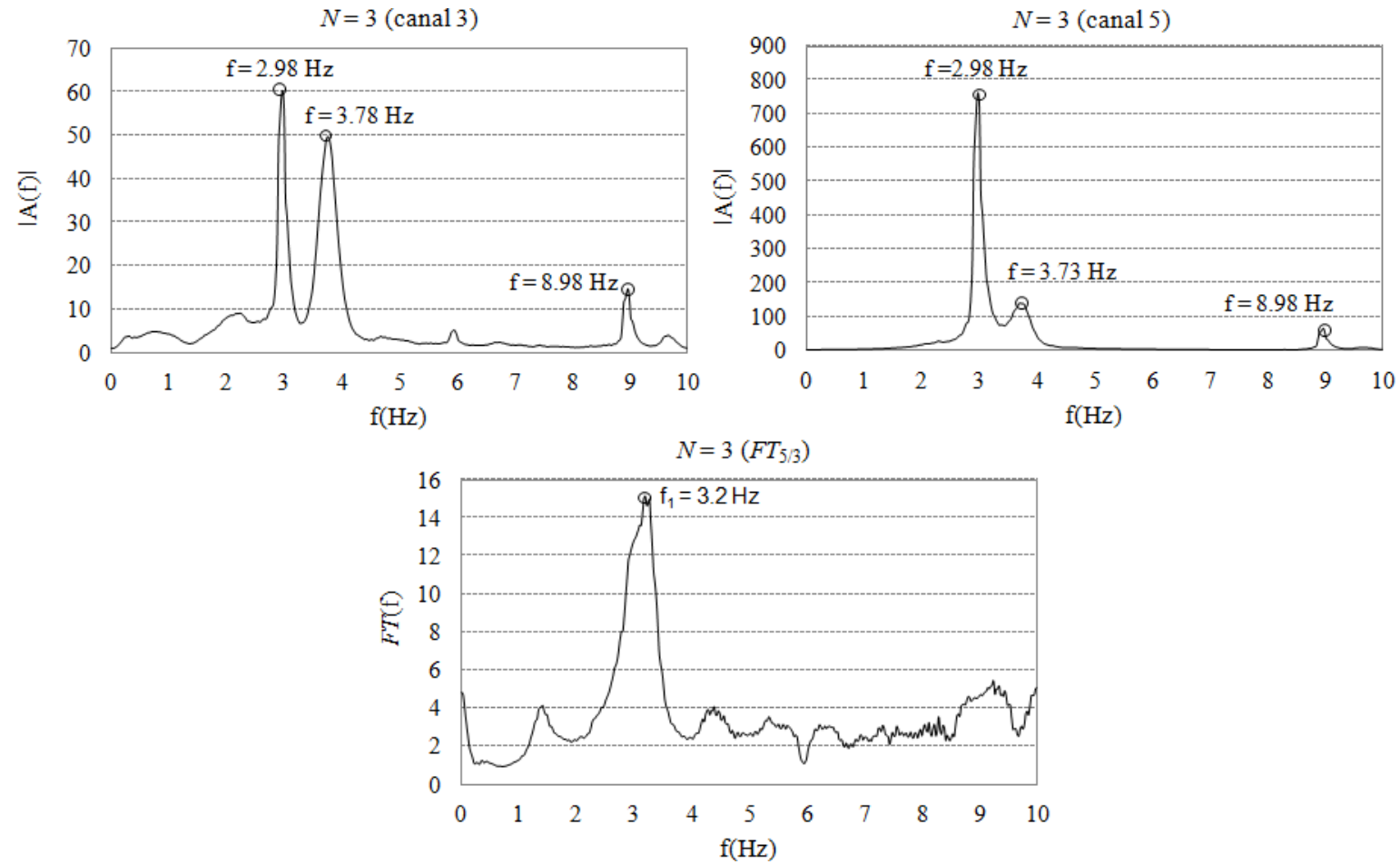

Figura 12. Espectros de amplitudes de Fourier de los canales 3 (entrada) y 5 (salida) con su correspondiente $F T$ obtenidos de una prueba de vibración forzada $(N=3)$

En las figuras 11 y 12 se pueden observar distintos picos en las gráficas de los espectros de amplitudes de Fourier, los cuales se asocian con las frecuencias del sistema completo (marco de concreto reforzado, péndulo de prueba y edificio del cual está colgado el péndulo). Más aún, en los espectros de Fourier de los canales 3 y 5 para la prueba de vibración forzada (figura 12), también se identifica la frecuencia de la excitación (cerca de $3 \mathrm{~Hz}$ ). De hecho, el péndulo de pruebas excita al marco de concreto con una señal que es la respuesta dinámica del sistema completo, correspondiente a la plataforma del péndulo.

Del análisis de ambas $F T$ (figuras 11 y 12), se observa que la primera frecuencia del marco podría identificarse como $f=3.2 \mathrm{~Hz}$ para esa etapa de daño $(N=3)$. Nótese que los puntos observados en los espectros de amplitudes de Fourier para los canales de entrada y salida, no necesariamente corresponden con las frecuencias modales del marco de concreto. En el caso de la prueba de vibración forzada, la frecuencia de excitación $(f=2.98 \mathrm{~Hz})$ aparece en los espectros de amplitudes de Fourier con una máxima amplitud, mientras que desaparece en la FT correspondiente (figura 12). En el caso de las pruebas de vibración ambiental, es usual considerar que la excitación corresponde a ruido blanco, cuyo espectro de amplitudes de Fourier se asemeja a una línea horizontal que representa similares amplitudes de Fourier para todas las frecuencias. En tal caso, las frecuencias modales del sistema completo podrían identificarse utilizando los espectros de amplitudes de Fourier.

En las pruebas llevadas a cabo, se observó que la hipótesis de ruido blanco para las pruebas de vibración ambiental no se cumplió, ya que aparecen distintos picos en las gráficas de los espectros de amplitudes de Fourier (canales de entrada y salida) que hacen ver que la excitación no corresponde con una señal de ruido blanco. Estos picos no deben considerarse como frecuencias modales del marco, ya que corresponden más bien a ruido en la señal de entrada. Es probable que la vibración del suelo en los 
alrededores del laboratorio durante las pruebas de vibración ambiental si se haya asemejado a una señal de ruido blanco, pero la aceleración registrada en la plataforma del péndulo, la cual fue la que en realidad excitó al marco de concreto, fue filtrada en cierta manera por el sistema edificio de laboratorio - péndulo de prueba. Este "filtrado" pudo haber modificado las características de la excitación del marco de concreto durante las pruebas de vibración ambiental, provocando una excitación diferente a ruido blanco.

La figura 13 muestra distintas $F T$ correspondientes a las pruebas de vibración forzada para distintos estados de daño del marco. En cada gráfica se señala la primera frecuencia modal del marco $f_{1}$, que corresponde a esa etapa de daño. Para el caso sin daño $(N=1)$, se observa que $f_{1}=3.2 \mathrm{~Hz}$. Este valor disminuyó en las pruebas siguientes, conforme fue creciendo el daño del marco. De acuerdo a la figura 8 , en la prueba $N=6$ se establece el límite del comportamiento elástico del marco, en cuyo caso se aprecia una disminución del valor de $f_{1}$, la cual pasó de $f_{1}=3.2 \mathrm{~Hz}$ para $N=1$, a $f_{1}=2.97 \mathrm{~Hz}$. Este decremento se puede atribuir al agrietamiento inicial del marco, el cual se detectó visualmente después de la prueba $N=$ 4. Para la prueba $N=7$, donde se considera que inicia la fluencia del marco, se observa una caída abrupta del valor de $f_{1}$, el cual pasa de $f_{1}=2.97 \mathrm{~Hz}(N=6)$ a $f_{1}=2.24 \mathrm{~Hz}(N=7)$. Para las demás pruebas $(N=8$ a $15)$, no se detecta un cambio importante en el valor de $f_{1}$, en comparación al valor identificado al inicio de la fluencia del marco $(N=7)$.

La figura 14 muestra la variación de $f_{1}$ en función de la etapa de daño $N$ y la máxima distorsión global del modelo $\gamma_{\max }$. Los resultados de las pruebas de vibración ambiental se muestran en la figura 14a y los de las pruebas de vibración forzada en la figura 14b. La variación se presenta en términos de la diferencia en porcentaje del valor de $f_{1}$ para cada etapa de carga (daño) en comparación al valor de $f_{1}$ identificado para el caso sin daño $(N=1)$. En la gráfica correspondiente a las pruebas de vibración forzada (figura 14a) se observan tres etapas de daño. La primera corresponde a valores de $N=1$ a 3 . En esta etapa se aprecia un comportamiento elástico del marco, ya que no se observan variaciones importantes del valor de $f_{1}$. En la segunda etapa $(N=4$ a 6$)$ se observa una degradación inicial de la rigidez lateral del marco debida a su agrietamiento inicial, el cual se detecto visualmente a partir de la prueba $N=4$. La mayor variación de $f_{1}$ durante la segunda etapa fue de $7 \%$ (aprox.). La tercera etapa corresponde a la fluencia del marco y comenzó a partir de la prueba $N=7$, donde se presentó una caída abrupta del valor de $f_{1}$ que representó una diferencia en porcentaje cercana al $30 \%$ en relación al valor de $f_{1}$ para el caso sin daño. A partir de la prueba $N=7$ no se aprecian cambios significativos en los valores de $f_{1}$ que se identificaron, no obstante que se alcanzaron demandas de ductilidad altas. Las tres etapas de comportamiento del marco que se aprecian en la figura 14a, corresponden con buena aproximación a las etapas de comportamiento que se identifican en la figura 8 .

Para las pruebas de vibración ambiental (figura 14a), la tendencia de la gráfica es similar a la que se observa para las pruebas de vibración forzada (figura 14b). Sin embargo, se observa una mayor dispersión en los resultados de las pruebas de vibración ambiental. Es importante tomar en cuenta, que este comportamiento se explica en la medida que en las pruebas de vibración ambiental no se alcanza a observar el efecto no lineal en el comportamiento del modelo originado por el trabajo de las grietas que se abren y cierran, mientras que en las pruebas de vibración forzada, sí se observa dicho efecto. En el caso de vibración ambiental, la diferencia de $f_{1}$ al comparar el inicio de la fluencia del marco respecto al caso sin daño fue menor que en el caso de vibración forzada.

De acuerdo a estos resultados, se observa que el cambio de la primera frecuencia modal del marco de concreto $f_{l}$, indica con buena aproximación el nivel de daño alcanzado en las distintas pruebas realizadas. Estos cambios de frecuencia permiten establecer si el modelo tuvo comportamiento elástico o inelástico, pero no permiten conocer la demanda de ductilidad que se alcanzó durante las pruebas. 

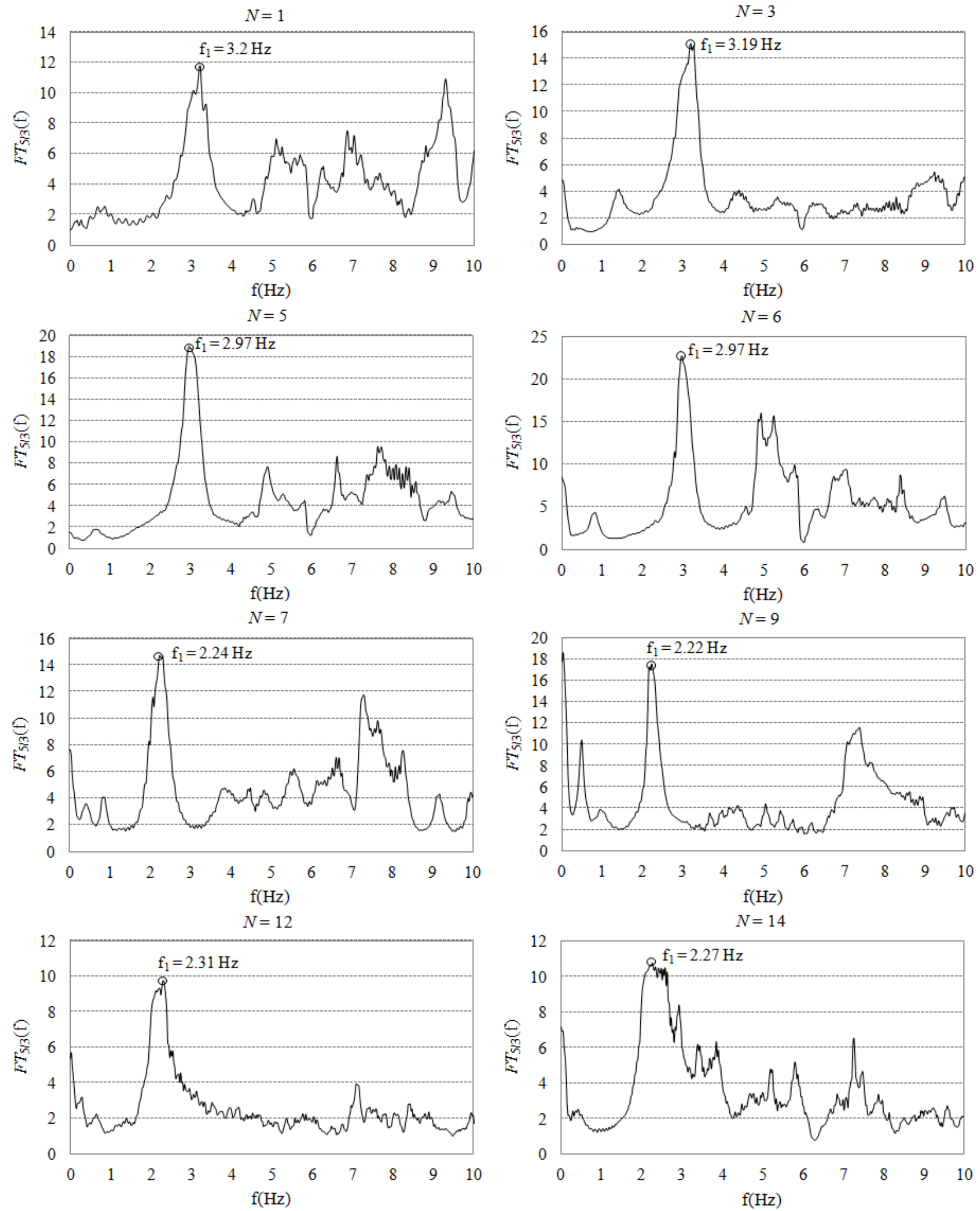

Figura 13. FT correspondientes a distintas etapas de daño (pruebas de vibración forzada) 


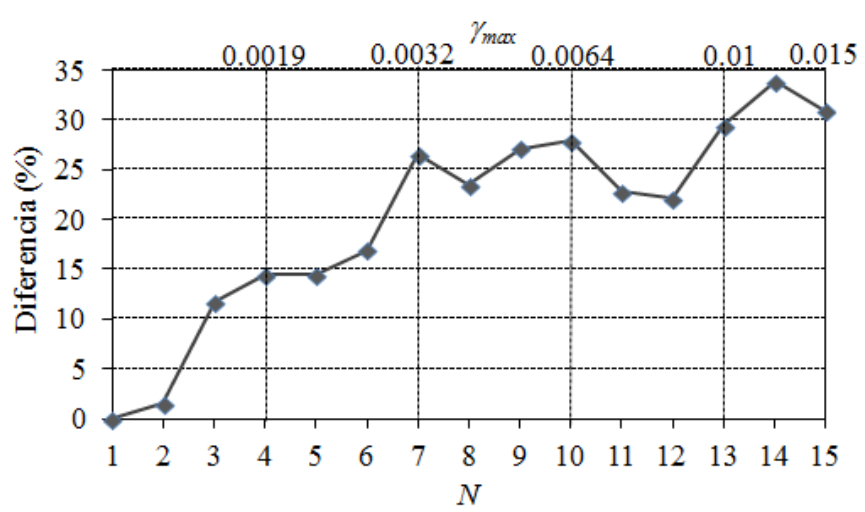

a) Vibración ambiental

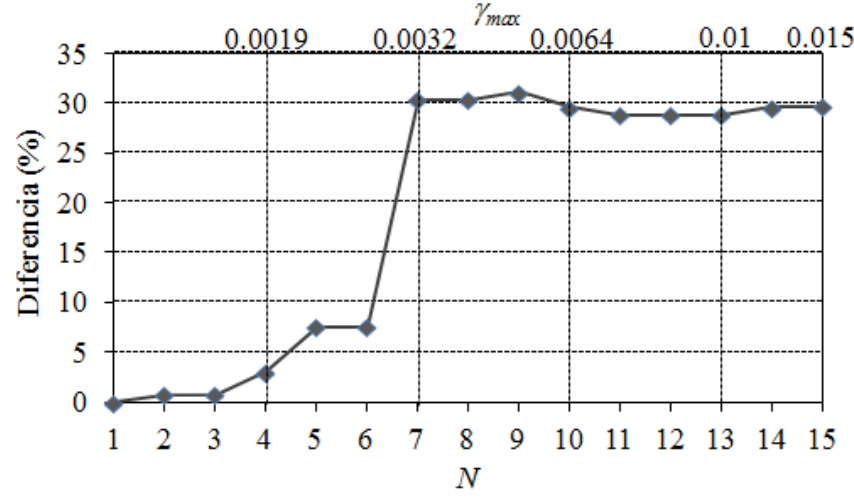

b) Vibración forzada

Figura 14. Variación de $f_{1}$ en función de $N$ y $\gamma_{\max }$ a) Pruebas de vibración ambiental b) Pruebas de vibración forzada

También se calcularon los valores MAC (Allemang y Brown, 1982) a partir de las formas modales correspondientes a $f_{l}$ que fueron identificadas en las distintas etapas de daño. Los valores MAC son un indicador de la correlación que hay entre dos modos de un sistema dinámico, por lo que en este caso sirvieron para analizar la correlación entre las formas modales asociadas a una etapa de daño en particular, en comparación a la forma modal correspondiente a la prueba $N=1$. En este caso los valores $M A C$ no fueron un buen indicador del daño en el modelo. Sus valores estuvieron muy próximos a 1 para casi todas las pruebas. Este resultado parece obvio, ya que el criterio $M A C$ se formuló para discriminar formas modales que correspondan a diferentes modos, sin embargo, este criterio se ha utilizado de manera generalizada para tratar de identificar el daño a partir de la comparación de dos formas modales que corresponden al mismo modo pero a dos etapas de daño diferentes.

En general, los cambios de las formas modales son más sutiles que los cambios de las frecuencias (Chang et al., 2003). Doebling et al. (1996) presentan algunos casos de aplicación de ambos criterios (frecuencias y formas modales). En dicho trabajo, se aprecia la dificultad para identificar el daño estructural utilizando frecuencias o formas modales, sobre todo para niveles de daño medio y bajo. En contraste, Trifunac et al. (2010) concluyen que el cambio de la primera frecuencia modal considerando el edificio que estudian empotrado en su base, es un buen indicador de daño, incluso para niveles bajos de comportamiento inelástico (daño incipiente). Los niveles de decremento de la primer frecuencia modal del marco de concreto que se estudia en este trabajo, resultaron muy similares a los reportados por Trifunac et al. (2010) correspondientes a su caso de estudio.

\section{FUNCIONES DE TRANSFERENCIA (FT)}

Las FT, también conocidas como funciones de respuesta en frecuencia $(F R F)$, han sido ampliamente utilizadas para detectar el daño de distintos sistemas estructurales (Roitman et al., 1992; Lew, 1995; Heylen et al., 1996; Wang et al., 1997; James et al., 1998; Thyagarajan et al., 1998; Sampaio et al., 1999; Zang et al., 2001; Lee et al., 2002; Zang et al., 2003; Ni et al., 2006; Li et al., 2011). Por ejemplo, algunos estudios han analizado el cambio de la curvatura de las $F R F$ como un parámetro de daño estructural (Sampaio et al., 1999), otros estudios (Li et al., 2011; Ni et al., 2006; Zang et al., 2001) han utilizado el análisis de los componentes principales de las $F R F$ comprimidas y redes neuronales para predecir el daño estructural. En general, se ha demostrado que el uso de las $F R F$ es una herramienta útil, 
confiable y robusta para detectar el daño estructural en modelos experimentales y analíticos, aún para etapas de daño inicial.

En este trabajo se analiza el coeficiente de Pearson $(r)$ como un posible indicador de daño. Para ello se calcula dicho coeficiente entre una $F T$ que corresponde al estado sin daño del modelo $\left(F T_{u}\right)$ y otra $F T$ correspondiente a un determinado nivel de daño del modelo $\left(F T_{d}\right)$. Como se sabe, el coeficiente de Pearson es un índice que se utiliza para medir la relación entre dos variables aleatorias, y varía de -1 a 1. Un valor de $r=1$ indica una correlación positiva perfecta entre ambas variables, es decir, que cuando una variable se incrementa la otra también lo hace en una proporción constante. Un valor de $r=0$ indica que no existe relación entre ambas variables. Esta condición no necesariamente representa que ambas variables sean independientes entre sí, ya que es posible que exista una relación no lineal entre ellas. Valores de $r$ entre 0 y 1 representan correlación positiva y muestran cierto nivel de dependencia entre ambas variables. Valores negativos de $r$ señalan que existe una correlación negativa, lo que significa que cuando una variable se incrementa la otra tiene un decremento. De acuerdo a estos conceptos, se esperaría que los valores de $r$ tiendan a 0 en la medida que la estructura se encuentre con daño. Valores de $r$ cercanos a 1 indicarían que existe poco daño en el marco que se probó, mientras que valores de $r$ próximos a 0 alertarían de la presencia de un alto nivel de daño estructural en el marco. Esto es obvio ya que se espera quelas $F T$ sean muy similares al comparar el estado sin daño del marco respecto a un estado con moderado daño, y a su vez, se espera que las $F T$ sean poco parecidas al comparar el estado sin daño del marco respecto a un estado con alto nivel de daño estructural.

El coeficiente de correlación se calculó con base en las siguientes expresiones

$r=\frac{S_{u d}}{\sqrt{S_{u u} S_{d d}}}$

donde

$S_{u u}=\sum_{i=1}^{m} F T_{u i}^{2}-\frac{\left(\sum_{i=1}^{m} F T_{u i}\right)^{2}}{m}$

$S_{d d}=\sum_{i=1}^{m} F T_{d i}^{2}-\frac{\left(\sum_{i=1}^{m} F T_{d i}\right)^{2}}{m}$

$S_{u d}=\sum_{i=1}^{m}\left(F T_{u i}\right)\left(F T_{d i}\right)-\frac{\left(\sum_{i=1}^{m} F T_{u i}\right)\left(\sum_{i=1}^{m} F T_{d i}\right)}{m}$

$m$ es el tamaño de la muestra de las FT (número de frecuencias que se consideran en el análisis estadístico).

El uso del coeficiente de Pearson en este trabajo, no supone que exista una relación lineal entre las FT para dos estados de daño del modelo; simplemente se utiliza para investigar si este parámetro puede asociarse con el nivel de daño de la estructura. Los resultados muestran que el coeficiente de Pearson puede ser útil para esta estimación, sin que esto represente un análisis estadístico formal.

Las figuras 15 y 16 muestran la variación del coeficiente de correlación $r$ para distintos pares de $F T$ en función de la etapa de daño del modelo $\left(\gamma_{\max }\right)$ y el número de prueba $N$. La figura 15 muestra los 
resultados de las pruebas de vibración forzada y la figura 16 los resultados de las pruebas de vibración ambiental. Las $F T$ se calcularon como el cociente de los espectros de amplitudes de Fourier correspondientes a los canales 3 y $5\left(F T_{5 / 3}\right)$ y 2 y $3\left(F T_{2 / 3}\right)$. Los canales de salida fueron el 5 localizado en el segundo nivel del marco y el 2 localizado en el primer nivel. El canal 3 que fue el canal de entrada se localizó en la plataforma del péndulo, es decir en la base del marco (figura 6). En todos los cálculos $F T_{u}$ correspondió a la prueba $N=1$, mientras que las $F T_{d}$ correspondieron a las distintas pruebas realizadas, desde $N=1$ hasta $N=15$. El ancho de banda de las $F T$ que se consideró en el cálculo de $r$, fue de 0.0244 $\mathrm{Hz}$ a $20 \mathrm{~Hz}(m=820$ muestras $)$.

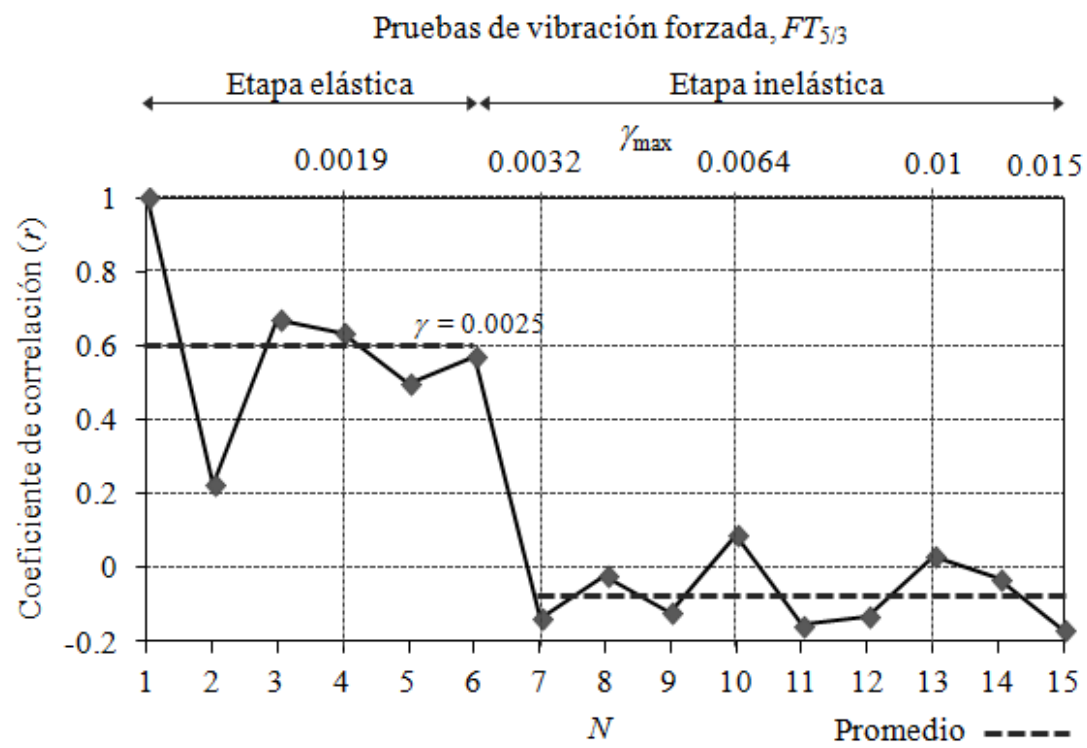

a) Gráfica correspondiente a $F T_{5 / 3}$

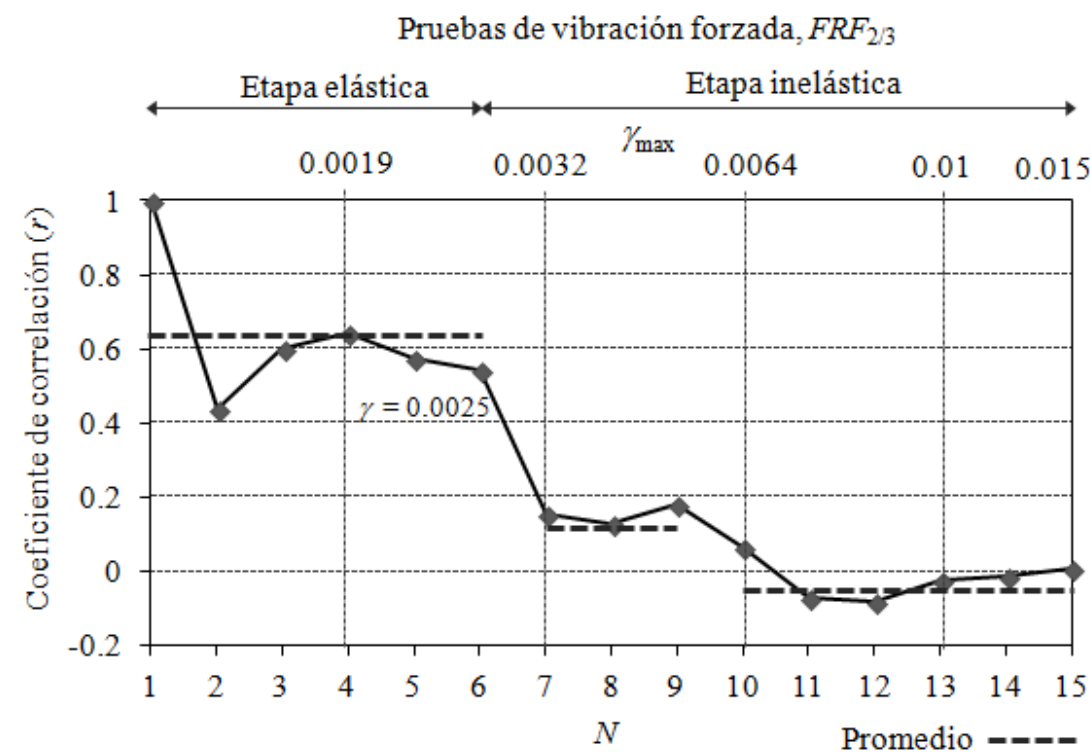

b) Gráfica correspondiente a $F T_{2 / 3}$

Figura 15. Variación del coeficiente de correlación $r$ en función de la etapa de daño $\gamma_{\max }$ y el número de prueba $N$ (pruebas de vibración forzada) 
Pruebas de vibración ambiental, $F T_{5 / 3}$

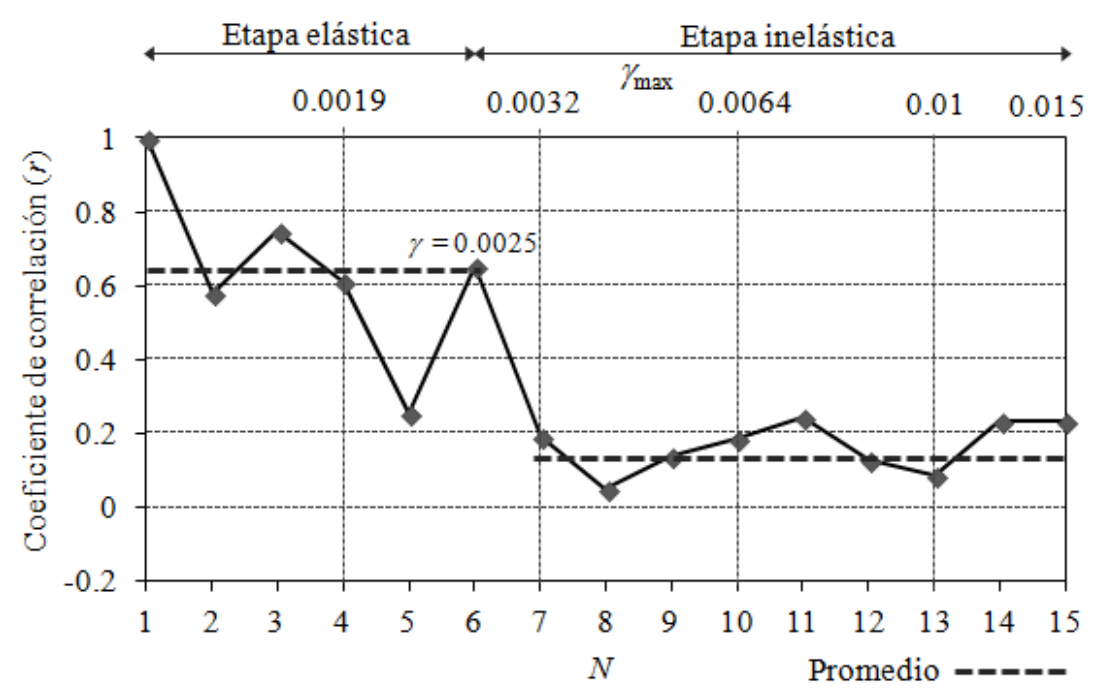

a) Gráfica correspondiente a $F T_{5 / 3}$

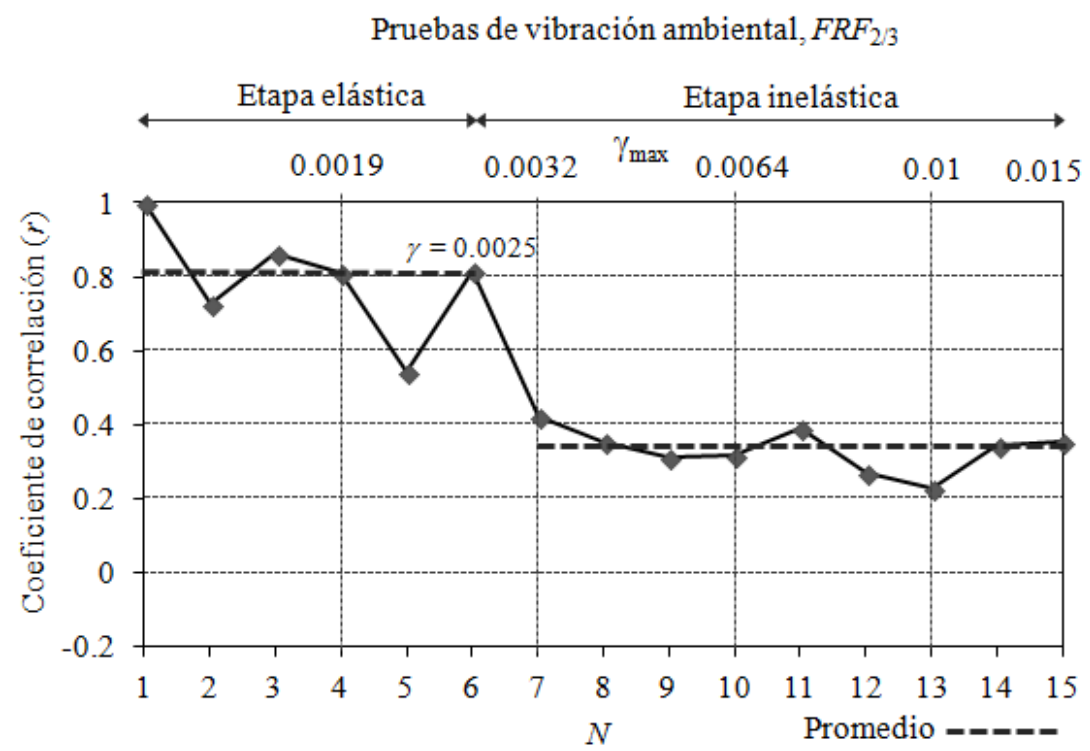

b) Gráfica correspondiente a $F T_{2 / 3}$

Figura 16. Variación del coeficiente de correlación $r$ en función de la etapa de daño $\gamma_{\max }$ y el número de prueba $N$ (pruebas de vibración ambiental)

Los resultados muestran que los valores de $r$ cambian considerablemente para $\gamma_{\max }>0.0025(N=6)$ en comparación a los que se obtienen para $\gamma_{\max }=<0.0025$. Tomando en cuenta que $\gamma_{\max }=0.0025$ representa el punto de fluencia del modelo, los resultados indican que la etapa de fluencia del modelo (intervalo inelástico) puede detectarse mediante el coeficiente de correlación $r$ entre las $F T$ obtenidas en distintas etapas de daño (elástica e inelástica). Este comportamiento es similar en ambos tipos de pruebas (vibración forzada y ambiental), así como para ambas FT ( $F T_{5 / 3}$ y $\left.F T_{2 / 3}\right)$. La Tabla 2 muestra el promedio de $r$ denotado como $\bar{r}$, considerando ambas etapas de comportamiento del modelo, elástica e inelástica. 
Los mayores valores de $\bar{r}$ en ambas etapas de comportamiento del modelo, elástica e inelástica, se alcanzaron durante las pruebas de vibración ambiental. Las menores diferencias entre los valores de $\bar{r}$ comparando las etapas elástica e inelástica, también se obtuvieron en las pruebas de vibración ambiental. De cualquier forma, estas diferencias fueron suficientes para distinguir entre ambos tipos de comportamiento (elástico e inelástico). En el caso de las pruebas de vibración forzada, las diferencias entre los valores de $\bar{r}$ que resultan al comparar el comportamiento elástico e inelástico del modelo fueron mayores que en el caso de vibración ambiental. En ambos tipos de prueba es evidente que $r$ tiende a cero cuando el daño del modelo se incrementa. La variación de $F T_{2 / 3}$ para el caso donde se analizan los resultados de las pruebas de vibración forzada, permitió identificar tres etapas de comportamiento del marco. Una que corresponde a su comportamiento elástico $(N=1$ a 6$)$ y otras dos que se presentan en la etapa de fluencia ( $N=7$ a 9$)$ y $(N=10$ a 15). La etapa que comprende a las pruebas $N=7$ a 9 se identifica con una demanda de ductilidad cercana a 2.0.

Tabla 2. Valores promedio del coeficiente de correlación $(\bar{r})$

\begin{tabular}{cccc}
\hline FT & Estado de daño & $\begin{array}{c}\text { Pruebas de vibración } \\
\text { forzada }\end{array}$ & $\begin{array}{c}\text { Pruebas de vibración } \\
\text { ambiental }\end{array}$ \\
\hline $\mathrm{FT}_{5 / 3}$ & Etapa elástica & 0.6 & 0.64 \\
& Etapa inelástica & -0.07 & 0.16 \\
$\mathrm{FT}_{2 / 3}$ & Etapa elástica & 0.63 & 0.79 \\
& Etapa inelástica & $0.13 \mathrm{y}-0.047^{*}$ & 0.33 \\
\hline
\end{tabular}

*Se identificaron tres estados de daño en este caso, uno en la etapa elástica y dos en la inelástica.

\section{CONCLUSIONES}

Se estudió en laboratorio un marco tridimensional de concreto reforzado al cual se le generaron distintas etapas progresivas de daño que simularon incrementos crecientes de la intensidad sísmica del movimiento del suelo. El marco se excitó mediante un péndulo de pruebas, el cual es un dispositivo que excitó el marco sujeto a su plataforma mediante un movimiento armónico horizontal a lo largo de un eje para frecuencias seleccionadas. Durante las pruebas se alcanzó una demanda de ductilidad máxima para la distorsión global del marco cercana a $\mu=6.0$. Para cada etapa de daño se hicieron pruebas de vibración ambiental y forzada, y se identificaron las propiedades dinámicas del marco. Fue posible caracterizar el nivel de daño del marco de concreto en términos de la correlación estadística entre pares de funciones de transferencia $(F T)$, obtenidas para la condición inicial sin daño del marco y etapas subsecuentes con determinados niveles de daño.

Con base en las pruebas experimentales realizadas y en la interpretación de sus resultados, las principales conclusiones de este trabajo son las siguientes:

1. Los valores que se obtuvieron del coeficiente de correlación $r$ entre pares de $F T$ correspondientes a dos estados diferentes de daño, sin daño $(N=1)$ y con daño $(N=2$ a 15$)$, soportan la idea de que $r$ es un parámetro útil para detectar el daño causado por fluencia. En este estudio se encontró que el valor promedio de $r$ que se obtiene al considerar las pruebas posteriores al inicio de la fluencia del modelo $(N=$ 7 a 15), resultó cercano a 0.0 para las pruebas de vibración forzada y a 0.16 para las pruebas de vibración ambiental. Para las pruebas realizadas antes de alcanzar el inicio de la fluencia del modelo $(N=1$ a 6$)$, el promedio de $r$ resultó de 0.6 en el caso de vibración forzada y de 0.64 en el de vibración ambiental. La diferencia entre los promedios de $r$ que se obtuvieron para las etapas elástica e inelástica del modelo, fueron lo suficientemente grandes en ambos casos (vibración ambiental y forzada) para identificar el daño estructural causado por la fluencia. Estos resultados corroboran las conclusiones de distintos autores, respecto al uso promisorio de las FT para detectar daño en diferentes sistemas estructurales. En este 
trabajo se propuso a $r$ como variable de decisión para detectar el daño estructural del modelo que se estudió.

2. En general, se observó que los valores de $r$ mayores a 0.6 no representan daño estructural causado por fluencia, mientras que valores de $r$ menores a 0.35 podrían alertar respecto a la aparición de daño estructural debido a la fluencia de la estructura. Estos límites deben manejarse con cuidado en estructuras similares al modelo que se estudió en este trabajo y en ningún caso deben extrapolarse a otro tipo de estructuras. Es importante destacar que $r$ fue capaz de detectar el daño estructural del modelo, aún para demandas de ductilidad bajas, es decir, daño incipiente. Ésta es una característica importante y deseable que deben tener los procedimientos de detección de daño estructural, para que sean confiables.

En este caso no fue posible identificar la demanda de ductilidad del modelo en función de $r$.

3. En el caso de los cambios de frecuencia, se observó que la primera frecuencia de traslación del modelo en la dirección $\mathrm{X},\left(f_{1}\right)$, experimentó cambios durante la etapa de comportamiento elástico del modelo menores a $15 \%$ en las pruebas de vibración ambiental, y a $7 \%$ en las pruebas de vibración forzada. Estos cambios no necesariamente representan daño estructural del modelo, si no que se asocian al agrietamiento inicial que se presentó en la etapa de comportamiento elástico del modelo. En ambos casos (pruebas de vibración ambiental y forzada), los cambios de $f_{l}$ durante el comportamiento inelástico del modelo alcanzaron valores cercanos al $30 \%$. Estos cambios de frecuencia fueron suficientes para poder distinguir entre ambos tipos de comportamiento del modelo (elástico e inelástico). Sin embargo, no fue posible identificar las demandas de ductilidad del modelo en función de estos cambios de frecuencias.

En general, es más recomendable el uso de las $F T$ que el de las frecuencias modales. Esto se debe a que las FT se obtienen directamente de los datos registrados, mientras que las frecuencias modales se extraen indirectamente mediante procedimientos de extracción modal que pudiesen contaminar en mayor medida los valores de las frecuencias que se obtengan, en comparación a la posible contaminación que pudiesen tener las $F T$. Por ello, se considera que las $F T$ son más confiables que las frecuencias modales para detectar el daño de las estructuras.

4. Los valores $M A C$ que se obtuvieron al comparar las formas modales con daño y sin daño correspondientes a la primera forma modal de traslación del modelo, resultaron próximos a 1 en todos los casos, sin importar el nivel de daño que se analizara. Por ello no fue posible identificar el daño estructural del modelo con este parámetro.

5. La reducción de la rigidez lateral del marco, respecto a su condición inicial $(N=1)$, fue cercana a $9 \%(N=6)$ durante la etapa de comportamiento "elástico". En la etapa "inelástica", está reducción varió de $40 \%(N=7)$ a $60 \%(N=15)$.

6. Se verificó el uso del péndulo de pruebas para llevar a cabo pruebas dinámicas en modelos estructurales de laboratorio. El péndulo de pruebas es un dispositivo sencillo y novedoso que permite inducir aceleraciones en la base a los modelos que se prueben. Los resultados confirman que fue posible inducir distintos niveles de daño al marco de concreto, relacionados con intensidades crecientes del movimiento de su base.

7. El uso de $r$ como variable de decisión al utilizar pruebas de vibración ambiental para detectar el daño por fluencia de una estructura causado por la ocurrencia de un temblor, parece ser un procedimiento simple, razonable y confiable; sobre todo en casos de emergencia. El cálculo de $r$ es simple en comparación con otros procedimientos de detección de daño, los cuales dependen de sofisticados 
algoritmos que se basan en una gran cantidad de datos obtenidos bajo condiciones especiales en lo referente a: localización y número de sensores, tipo de excitación, etc.

Las conclusiones generales de este trabajo son parcialmente válidas para estructuras formadas por marcos de concreto reforzado (sin muros confinados o arriostramientos), con un modo de falla a flexión gobernado por la formación de articulaciones plásticas en las trabes. Es decir, estructuras con trabes débiles y columnas fuertes.

Para aplicar un procedimiento de detección de daño estructural que se base en $r$, se requieren conocer las $F T$ correspondientes al estado sin daño de la estructura. Por ello es importante realizar pruebas de vibración ambiental, tanto en las estructuras nuevas como en las estructuras existentes que se consideren sanas, para establecer una referencia que sirva en una futura identificación de daño estructural.

Bajo las condiciones actuales es difícil realizar mediciones de vibración en la mayoría de las estructuras convencionales, debido fundamentalmente al costo de los equipos de medición y a las dificultades técnicas de las pruebas. Sin embargo, este tipo de pruebas son fundamentales en los distintos procedimientos de detección de daño, como el que aquí se presenta. De ahí la necesidad que se tiene de desarrollar técnicas y equipos de medición de bajo costo que permitan hacer las pruebas dinámicas de manera rutinaria en la mayoría de las construcciones. No basta con desarrollar procedimientos dinámicos de detección de daño que sean cada vez más confiables, también se requiere hacer factible la realización de pruebas de vibración bajo los estándares y requerimientos que demandan los procedimientos desarrollados. Se espera que en un futuro las normas y reglamentos de construcción se encarguen de regular los procedimientos de detección de daño y las pruebas que se requieran, lo cual permitirá poner en práctica muchos de los métodos dinámicos de detección de daño que se han desarrollado.

También es importante realizar estudios de diferentes modelos de edificios, analíticos y experimentales, para corroborar los resultados obtenidos en este trabajo.

\section{REFERENCIAS}

Allemang, R J y D L Brown (1982), “A correlation coefficient for modal vector analysis”, Proceedings of the 1st international modal analysis conference, Orlando, Florida, USA.

Chang, P C, A Flatau y S C Liu (2003), "Review Paper: Health Monitoring of Civil Infrastructure", Structural Health Monitoring, An International Journal, Vol. 2, No. 3, pp. 257-267.

Chiu, H CH (1997), "Stable base line correction of digital strong - motion data", Bull. of the Seismological Society of America, Vol. 87, No. 4, pp. 932-944.

Clough, R W y J Penzien (1993), Dynamics of structures, McGraw - Hill, Singapore.

De la Colina, J y J Valdés (2010), "Péndulo de prueba para el estudio dinámico de modelos estructurales", Revista de Ingeniería Sísmica, Vol. 82, pp. 35-56.

Doebling, S W, C R Farrar, M B Prime y D W Shevitz (1996), "Damage identification and health monitoring of structural and mechanical systems from changes in their vibration characteristics: a literature review", Report No. LA-13070-MS, Los Alamos National Laboratory, Los Alamos, USA.

Heylen, W y S Lammens (1996), "FRAC: a consistent way of comparing frequency response functions". Proceedings of conference on identification in engineering systems. 
James III, G H, D C Zimmerman y R L Mayes (1998), “ Experimental study of frequency response function (FRF) based damage assessment tools", Proceedings of the 16th International Modal Analysis Conference, Santa Barbara, CA, pp. 151-157.

Lee, U y J Shin (2002), “A frequency response function-based structural damage identification method", Computers and Structures, Vol. 80, pp. 117-132.

Lew, J S (1995), "Using transfer function parameter changes for damage detection of structures", AIAA Journal, Vol. 33, pp. 2189-2193.

Li, J, U Dackermann, Y Xu y B Samali (2011), "Damage identification in civil engineering structures utilizing PCA-compressed residual frequency response functions and neural network ensembles", Journal of Structural Control and Health Monitoring, Vol. 18, pp. 207 - 226.

Martínez, E y M Wakabayashi (1990), Diseño de estructuras sismorresistentes, McGraw-Hill, México.

Ni, Y Q, X T Zhou y J M Ko. (2006), "Experimental investigation of seismic damage identification using PCA-compressed frequency response functions and neural networks", Journal of Sound and Vibration, Vol. 290, pp. 242-263.

NTCC-2004 (2004), "Normas Técnicas Complementarias para Diseño y Construcción de Estructuras de Concreto",Gaceta Oficial del Distrito Federal, octubre.

Prine, D (1998), "Steel bridge retrofit evaluation", Proceedings of SPIE - The International Society for Optical Engineering, pp. 442-450.

Rhazi, J (2006), "Evaluation of concrete structures by the acoustic tomography technique", Structural Health Monitoring, An International Journal, Vol. 5, No. 4, pp. 333-342.

Roitman, N, P E Viero, C Magluta, R C Batista y L F L Rosa (1992), "Identification of offshore platform structural damage using modal analysis techniques", Mechanical Systems and Signal Processing, Vol. 6, pp. 287-295.

Salawu, O y C Williams (1995), "Bridge assessment using forced-vibration testing", Journal of Structural Engineering, Vol. 121, No. 2, pp. 161-173.

Sampaio, R P C, N M M Maia y J M M Silva (1999), "Damage detection using the frequency-responsefunction curvature method", Journal of Sound and Vibration, Vol. 226, pp. 1029-1042.

Thyagarajan, S K, M J Schulz, P F Pai y J Chung (1998), "Detecting structural damage using frequency response functions", Journal of Sound and Vibration, Vol. 210, pp. 162-170.

Trifunac, M D, M I Todorovska, M I Manic y B Bulajic (2010)."Variability of the fixed-base and soilstructure system frequencies of a building - The case of Borik-2 building", Journal of Structural Control and Health Monitoring, Vol. 17, pp. 120-151.

Voigt, T, Y Akkaya y S Shah (2003a), "Determination of early age mortar and concrete strength by ultrasonic wave reflections", Journal of Materials in Civil Engineering, Vol. 15, No. 3, pp. 247254.

Voigt, T y P Shah (2003b), "Nondestructive monitoring of setting and hardening of Portland cement mortar with sonic methods", Proceedings of the Sixth International Symposium on Non-Destructive Testing in Civil Engineering (NDT-CE 2003). Berlin, Germany.

Wang, Z, R M Lin y M K Lim (1997), "Structural damage detection using measured FRF data", Computer Methods in Applied Mechanics and Engineering, Vol. 147, pp. 187-197.

Wang, H (2004), "Theoretical evaluation of embedded plate-like and solid cylindrical concrete structures with guided waves", Ph.D. Thesis, Northwestern University. 
Zang, C y M Imregun (2001), “ Structural Damage Detection Using Artificial Neural Networks and Measured FRF Data Reduced Via Principal Component Projection, Journal of Sound and Vibration, Vol. 242, pp. 813-827.

Zang, C, M I Friswell y M Imregun (2003), "Structural Health Monitoring and Damage Assessment Using Measured FRFs from Multiple Sensors, Part I: The Indicator of Correlation Criteria. Key Engineering Materials, Vol. 245-246, pp. 131-140. 